\title{
The dimer-dependent catalytic activity of RAF family kinases is revealed through characterizing their oncogenic mutants
}

\author{
Jimin Yuan ${ }^{1}$ Wan Hwa $\mathrm{Ng}^{1} \cdot$ Paula Y. P. Lam ${ }^{1,2} \cdot$ Yu Wang ${ }^{1} \cdot$ Hongping Xia ${ }^{1} \cdot$ Jiajun Yap ${ }^{1}$ Shou Ping Guan ${ }^{1}$ \\ Ann S. G. Lee ${ }^{3,4,5} \cdot$ Mei Wang ${ }^{2} \cdot$ Manuela Baccarini $\mathbb{1}^{6}$ - Jiancheng $\mathrm{Hu}^{1,2}$
}

Received: 23 November 2017 / Revised: 18 May 2018 / Accepted: 21 May 2018 / Published online: 21 June 2018

(c) The Author(s) 2018. This article is published with open access

\begin{abstract}
Although extensively studied for three decades, the molecular mechanisms that regulate the RAF/MEK/ERK kinase cascade remain ambiguous. Recent studies identified the dimerization of RAF as a key event in the activation of this cascade. Here, we show that in-frame deletions in the $\beta 3-\alpha \mathrm{C}$ loop activate ARAF as well as BRAF and other oncogenic kinases by enforcing homodimerization. By characterizing these RAF mutants, we find that ARAF has less allosteric and catalytic activity than the other two RAF isoforms, which arises from its non-canonical APE motif. Further, these RAF mutants exhibit a strong oncogenic potential, and a differential inhibitor resistance that correlates with their dimer affinity. Using these unique mutants, we demonstrate that active RAFs, including the BRAF(V600E) mutant, phosphorylate MEK in a dimer-dependent manner. This study characterizes a special category of oncogenic kinase mutations, and elucidates the molecular basis that underlies the differential ability of RAF isoforms to stimulate MEK-ERK pathway. Further, this study reveals a unique catalytic feature of RAF family kinases that can be exploited to control their activities for cancer therapies.
\end{abstract}

\section{Introduction}

The Ras/rapidly-accelerated fibrosarcoma (RAF)/mitogenactivated protein kinase (MEK)/Extracellular signalregulated kinase (ERK) signaling plays a crucial role in

Electronic supplementary material The online version of this article (https://doi.org/10.1038/s41388-018-0365-2) contains supplementary material, which is available to authorized users.

Jiancheng $\mathrm{Hu}$

hu.jiancheng@nccs.com.sg

1 Division of Cellular and Molecular Research, Singapore, Singapore

2 Cancer and Stem Cell Program, Duke-NUS Medical School, 8 College Road, 169857 Singapore, Singapore

3 Division of Medical Sciences, National Cancer Centre Singapore, 11 Hospital Drive, Singapore 169610, Singapore

4 Office of Clinical \& Academic Faculty Affairs, Duke-NUS Medical School, 8 College Road, Singapore 169857, Singapore

5 Department of Physiology, National University of Singapore, 2 Medical Drive, 117597 Singapore, Singapore

6 Max F. Perutz Laboratories, University of Vienna, Doktor-BohrGasse 9, 1030 Vienna, Austria cell proliferation, survival, and differentiation [1, 2]. Aberrant activation of this kinase cascade causes developmental disorders and cancers [3-5]. Genetic alterations that hyperactivate the RAF/MEK/ERK kinase cascade exist in $>40 \%$ of cancers. To target this kinase cascade for cancer therapy, both RAF inhibitors and MEK inhibitors have been developed and applied to clinical treatment [6-8]. Unfortunately, their efficacy is limited by either intrinsic or rapidly acquired resistance. Understanding the regulation of the RAF/MEK/ERK kinase cascade could help us to design strategies to circumvent this resistance and develop more effective inhibitors for cancer treatment.

The RAF kinases CRAF, BRAF, and ARAF are a core component of the RAF/MEK/ERK kinase cascade. Dimerization among RAF isoforms is a key event in triggering the RAF/MEK/ERK kinase cascade [9-18], which not only turns on the kinase activity of RAF but also facilitates the activation of MEK by RAF [19]. Mechanistic studies have shown that the two protomers play distinct roles in RAF dimers: one functions as an allosteric activator to facilitate the assembly of an active conformation in the other, which acts as a receiver to catalyze the phosphorylation of substrates [20]. Distinct molecular traits between BRAF and CRAF result in their differential ability to turn on the RAF/ MEK/ERK kinase cascade by dimerization-driven 
transactivation [21]. Whether ARAF can be activated by dimerization and its role in this process are unclear.

The dimerization of RAF kinase occurs in both physiological and pathological conditions, which can be induced by active Ras [13, 17], inhibitor binding [9, 11, 12, 14], gene fusions [22-25], and alternative splicings [26]. Active Ras-induced homo/hetero-dimerization of RAF kinase is responsible not only for the pathway activation by physiological agonists but also for its hyperactivation by genetic alterations upstream of RAF in carcinogenesis [13, 17, 27]. Active Ras-induced RAF dimerization can be further enhanced by RAF inhibitors, which accounts for the paradoxical effect of RAF inhibitors in cancer therapy [11, 12, 14]. It has been speculated that the RAF kinases have a close conformation in which the N-terminus docks on the carboxyl-terminal kinase domain [28]. Active Ras binds to the N-terminus of RAF kinases, which breaks their close conformation and thus facilitates their dimerization via kinase domain. On the other hand, RAF inhibitors could alter the conformation of RAF kinase domain once loaded, which not only creates a dimer-favored conformation but also relives the inhibitory interaction between $\mathrm{N}$-terminus and kinase domain [29]. The inhibitory effect of $\mathrm{N}$-terminus on RAF dimerization could also be abolished by gene fusions or alternative splicing of mRNA. Chromosome translocations that lead to fusions of variable genes to the kinase domain of BRAF or CRAF have been extensively reported in cancers [22-25], while the alternative splicings that partially delete the N-terminus of BRAF(V600E) and thus enhance the dimerization of BRAF(V600E) isoforms have been shown as one of important mechanisms that underlie RAF inhibitor resistance in cancer therapy [26]. Recently, some RAF mutants (ARAF and BRAF) with inframe deletions in the $\beta 3-\alpha C$ loop have been reported as potential oncogenic drivers [30-34], although whether they are activated through enhanced dimerization remains unknown [30, 31, 33] or controversial [32, 34].

In this study, we characterized the RAF mutants with inframe deletions in the $\beta 3-\alpha \mathrm{C}$ loop, and found that both ARAF and BRAF mutants were activated by enhanced dimerization. Further, we showed that the limited allosteric and catalytic activities of ARAF arose from its noncanonical APE motif that leads to a lower propensity of dimerization in contrast to BRAF and CRAF. Finally, we used active RAF mutants with different dimerization properties as an efficient tool to investigate whether the dimerization of RAF after activation is required for its catalytic activity and demonstrated that active RAFs including BRAF(V600E) phosphorylated MEK in a dimerdependent manner. Our data clarifies how in-frame $\beta 3-\alpha \mathrm{C}$ loop deletions trigger RAF family kinases, reveals the molecular basis underlying the differential ability of RAF isoforms to stimulate MEK-ERK signaling, and illustrates a key step in the activation of the RAF/MEK/ERK kinase cascade.

\section{Results}

\section{Deletion of Q347A348 activates ARAF by enforcing dimerization}

By virtue of its apparent low activity and rare mutations in cancer genomes, the molecular mechanism regulating ARAF and its role in oncogenesis are ill-defined. Recently, Nelson et al. identified an active ARAF mutant with Q347A348 deletion and F351L conversion in patients with Langerhans cell histiocytosis [31]. To confirm this finding and to decipher the molecular basis of ARAF activation by this compound mutation, we expressed wild-type ARAF, and its Q347A348del mutants ( $\triangle Q A)$ with or without F351L mutation in 293T cell, and found that Q347A348del mutant was able to stimulate the MEK-ERK pathway independent of F351L status (Fig. 1a), suggesting a dominant role of the Q347A348 deletion in the activation of ARAF. Similar to other constitutively-active mutants of RAF kinases, a co-expression of a dominant-negative RAS mutant (N17RAS) with $\operatorname{ARAF}(\Delta \mathrm{QA})$ did not affect its activity in 293T cells (Fig. 1b), indicating that ARAF $(\Delta \mathrm{QA})$ is a constitutively-active mutant and does not require upstream stimuli to trigger its catalytic activity. This notion was further validated by the finding that a stable expression of $\operatorname{ARAF}(\triangle \mathrm{QA})$ in wild-type, $\mathrm{BRAF}-/-$, and CRAF-/ - fibroblasts activated the MEK-ERK pathway and transformed cells independent of endogenous RAF molecules (Figs. S1A and 1C). Moreover, the shRNAmediated knockdown of either CRAF in BRAF-/- fibroblasts or BRAF in CRAF-/- fibroblasts had no effect on the ability of $\operatorname{ARAF}(\triangle \mathrm{QA})$ to stimulate downstream signaling (Figure S1B).

Dimerization of RAF molecules is critical for their activation under variable conditions [9-17]. We thus investigated whether $\triangle \mathrm{QA}$ activates ARAF by enhancing dimerization. To do this, we carried out a complementary split luciferase assay [35] to measure the dimer affinity of ARAF and its $\Delta$ QA mutant. In this assay, the N-terminus and C-terminus of luciferase (hitherto referred to as Nluc and Cluc) were fused to ARAF and co-expressed in cells. RAF dimerization brings the Nluc and Cluc together and reconstitutes the luciferase activity. Thus, the luciferase activity correlates with the amount of dimerized ARAF. As shown in Fig. 1d, the treatment with RAF inhibitor Vemurafenib, an agonist of RAF dimerization, increased the luciferase activity of $293 \mathrm{~T}$ cells co-expressing ARAF-Nluc and ARAF-Cluc. In contrast, the 293T cells co-expressing $\operatorname{ARAF}(\Delta Q A)-N l u c$ and $\operatorname{ARAF}(\Delta Q A)$-Cluc showed a 

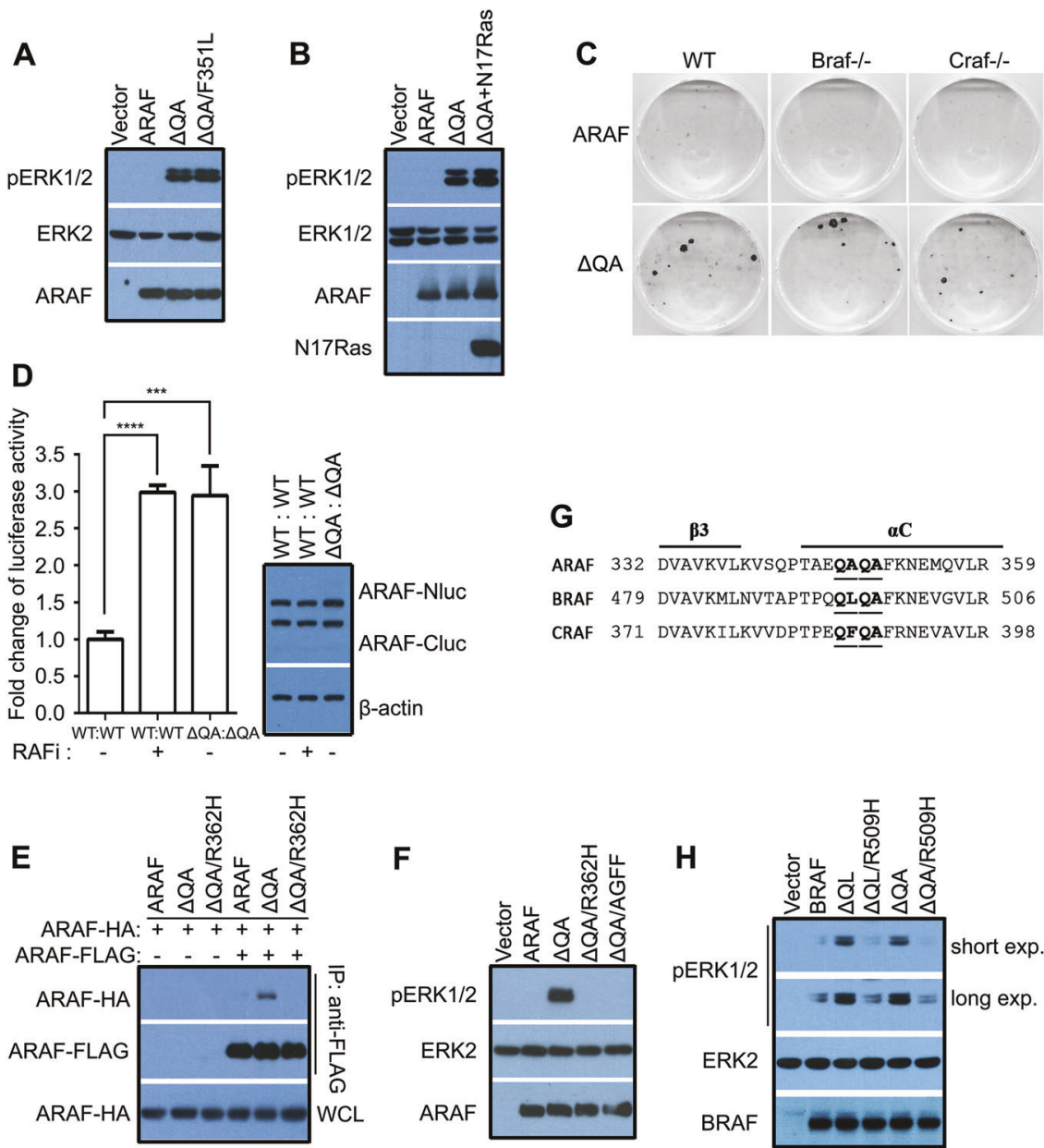

Fig. 1 The Q347A348 deletion activates ARAF by enhancing homodimerization. a $\operatorname{ARAF}(\triangle \mathrm{QA})$ and $\operatorname{ARAF}(\Delta \mathrm{QA} / \mathrm{F} 351 \mathrm{~L})$ have equal activity. The activity of ARAF mutants in $293 \mathrm{~T}$ transfectants was measured by anti-phospho-ERK1/2 immunoblot. b The activity of $\operatorname{ARAF}(\Delta \mathrm{QA})$ does not depend on upstream stimuli. $\operatorname{ARAF}(\Delta \mathrm{QA})$ was coexpressed with N17Ras in 293T cells, and its activity was measured as in a. c $\operatorname{ARAF}(\triangle \mathrm{QA})$ has a strong transforming ability independent of endogenous RAF molecules. Foci formation assay of immortalized fibroblasts expressing $\operatorname{ARAF}(\triangle \mathrm{QA})$ was carried out as described before [36, 37]. d, e $\operatorname{ARAF}(\Delta \mathrm{QA})$ has an elevated propensity to form homodimers. $\mathbf{d}$ The dimer affinity of $\operatorname{ARAF}(\Delta \mathrm{QA})$ was measured by using complementary split luciferase assay [35]. The dimerization of wild-type ARAF induced by 10 um Vemurafenib served as a positive control $(n=5, * * * p<0.001, * * * * p<0.0001)$. e The dimerization of $\operatorname{ARAF}(\Delta \mathrm{QA})$ was evaluated by co-immunoprecipitation assay. ARAF

constitutive luciferase activity comparable with that induced by Vemurafenib in wild-type ARAF transfectants, suggesting that $\operatorname{ARAF}(\triangle \mathrm{QA})$ mutant has an elevated ability to form homodimers. The homodimeriztion of $\operatorname{ARAF}(\Delta \mathrm{QA})$ in 293T cells can be further verified by coimmunoprecipitation assay. In contrast to its wild-type counterpart, HA-tagged $\operatorname{ARAF}(\Delta \mathrm{QA})$ could be pulled down by its FLAG-tagged version when co-expressed in 293T cells although the amount is a little (Fig. 1e). Using the same method, we next evaluated the ability of ARAF mutants with FLAG- or HA-tag were coexpressed in 293T cells, and immunoprecipitated by anti-FLAG beads and detected by anti-HA immunoblot. To exclude the effect of ERK1/2-mediated feedback on ARAF dimerization, all 293T transfectants in $\mathbf{d}$ and e were pretreated with $20 \mathrm{um}$ Tramentinib for $1 \mathrm{~h}$ before measurements. $\mathbf{f} \operatorname{ARAF}(\Delta \mathrm{QA})$ is activated by dimerization-driven transactivation. Mutations that disrupt the dimer interface $(\mathrm{R} 362 \mathrm{H})$ or block NtA-phosphorylation (AGFF) abolish the activity of $\operatorname{ARAF}(\triangle \mathrm{QA})$. The activity of ARAF mutants in 293T transfectants was measured as in $\mathbf{a} . \mathbf{g}, \mathbf{h}$ Homologous deletions activate BRAF in a dimer-dependent manner. $g$ The sequence alignment of human ARAF, BRAF, and CRAF reveals conserved residues in the $\beta 3-\alpha \mathrm{C}$ loop. $\mathrm{H}$, the activity of BRAF mutants in $293 \mathrm{~T}$ transfectants was measured as in a. All images are representative of at least three independent experiments

( $\triangle \mathrm{QA}$ ) to heterodimerize with BRAF, CRAF, and its wildtype counterpart, and found that it barely dimerized with these molecules (Figure S1C).

It has been reported that the dimerization-driven transactivation of RAF molecules might require the interaction of the negatively charged $\mathrm{N}$-terminal acidic motif (NtA motif) with the RKTRH motif in the $\alpha \mathrm{C}$-helix- $\beta 4$ loop of the other protomer, and mutations that abrogate the negative charge of the NtA motif or disrupt dimer interface block this process [20]. To determine whether $\operatorname{ARAF}(\Delta \mathrm{QA})$ is 


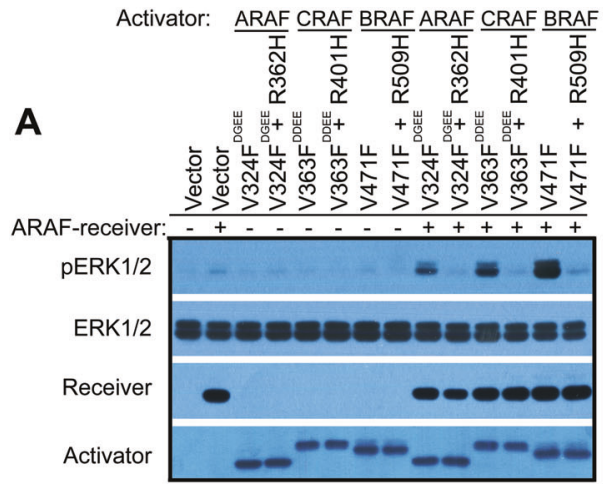

Fig. 2 ARAF has both allosteric and catalytic activities albeit less than BRAF and CRAF. a As a receiver, ARAF is strongly activated by BRAF, intermediately by CRAF, and weakly by itself through dimerization. The RAF co-activation assays were carried out as before $[20,37]$. Briefly, the activator and the receiver were co-expressed in

activated through a dimerization-driven transactivation, we mutated its NtA motif (SGYY to AGFF) or its central residue in the dimer interface $(\mathrm{R} 362 \mathrm{H})$, and found that both alterations impaired its activity (Fig. 1f), providing additional evidence that deletion of Q347A348 activates ARAF by enhancing homodimerization.

\section{Homologous deletions of two residues activate BRAF in dimer-dependent manner}

Since the Q347A348del activates ARAF by enhancing homodimerization and these two residues are conserved in the $\beta 3-\alpha C$ loop across all RAF isoforms (Fig. 1g), we next asked whether a homologous deletion would activate other RAF isoforms. As shown in Fig. 1h, BRAF mutants with a deletion of either Q494L495 or Q496A497 in the $\beta 3-\alpha C$ loop stimulated the MEK-ERK pathway when expressed in $293 \mathrm{~T}$ cells, and the central RH alteration (R509H) in the dimer interface abolished their activity. This suggests that BRAF can also be activated by the $\beta 3-\alpha \mathrm{C}$ loop deletiondriven homodimerization.

\section{ARAF has both allosteric and catalytic activities at lower levels than those of BRAF and CRAF}

The fact that ARAF signaling through ERK was activated by Q347A348 deletion-driven homodimerization indicated that it is able to function as both allosteric activator and receiver. To confirm this, we carried out a set of RAF coactivation assays [20, 36, 37]. In these assays, ARAF (AGFF) is a N-terminal truncated mutant (aa285-606) with a non-phosphorylatable NtA motif that functions as a receiver, whereas $\mathrm{ARAF}(\mathrm{DGEE} / \mathrm{V} 324 \mathrm{~F})$ is a similar truncatant with an acidic NtA motif and a fused catalytic spine $[38,39]$ that mimics the inhibitor-bound status and works as a kinase-dead allosteric activator (Table S1). When co-

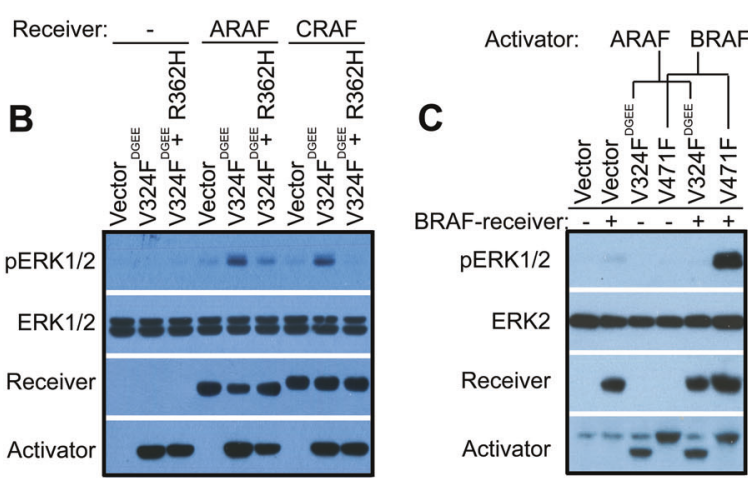

293T cells, and the phospho-ERK1/2 was measured by immunoblot. b. c As an activator, ARAF stimulates moderately the catalytic activity of ARAF and CRAF receivers, albeit hardly that of BRAF receiver. The RAF co-activation assays were carried out as in a. All images are representative of at least three independent experiments

expressed in 293T cells with allosteric activators derived from different RAF isoforms, ARAF(AGFF) was strongly activated by BRAF, intermediately by CRAF, and weakly by itself through dimerization (Fig. 2a). On the other hand, ARAF(DGEE/V324F) stimulated moderately the catalytic activity of ARAF and CRAF receivers, but only slightly that of BRAF receiver when co-expressed in $293 \mathrm{~T}$ cells (Fig. 2b, c). Taken together, these data suggest that ARAF can function as both receiver and allosteric activator, albeit less efficiently than the other two isoforms.

\section{The weak activity of ARAF arises from its non- canonical APE motif that decreases its dimer affinity}

Regulatory spine (R-spine) is a hallmark signature of active protein kinases that comprised of four conserved residues, namely RS1-4 [38, 39]. To explore the molecular basis underlying the weak activity of ARAF, we examined whether the R-spine-favored mutation could turn ARAF into a constitutively active kinase independent of dimerization-driven transactivation, as it has been shown for CRAF and BRAF [20,36, 38-41]. Similar to BRAF and CRAF, an ARAF mutant combining a RS3 mutation (L358M) and a negatively charged NtA motif showed a strong activity toward MEK-ERK pathway when expressed in 293T cells (Fig. 3a). Although the activity of this mutant (ARAF, DGEE/L358M) did not depend on the activation loop (AL)-phosphorylation, it was impaired by the central RH alteration (R362H) in the dimer interface (Fig. 3b), indicating again that ARAF has different characteristics from BRAF and CRAF. By aligning the kinase domain sequences of RAF isoforms, we found that ARAF had a non-canonical APE motif whose Pro is altered into Ala (Fig. $3 c)$. The APE motif localizes at the N-terminus of $\alpha$-helix $\mathrm{EF}(\alpha \mathrm{EF})$, and the Pro is a helix breaker that makes the Nterminus of $\alpha \mathrm{EF}$ flexible. The Glu (E) next to Pro in the 

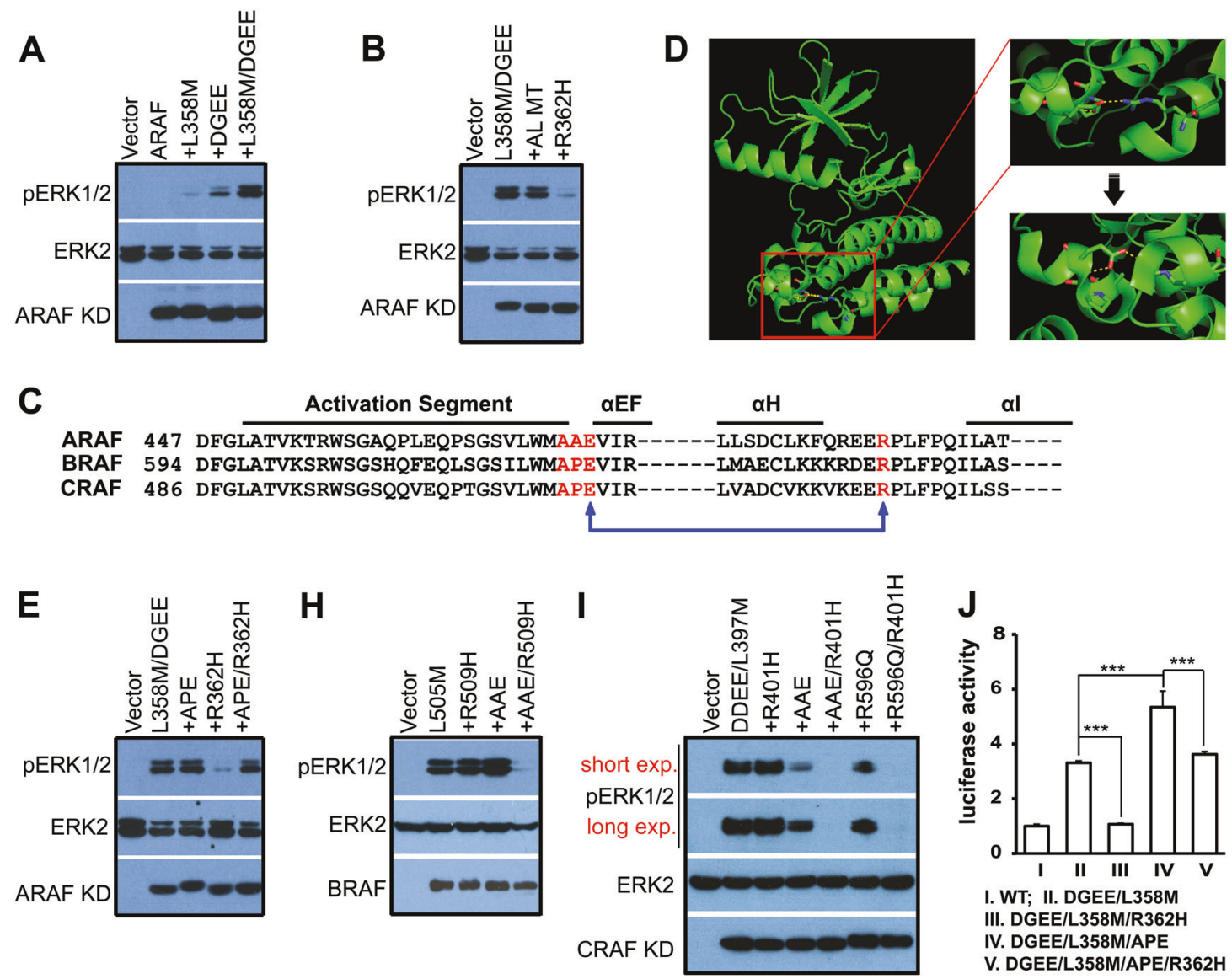
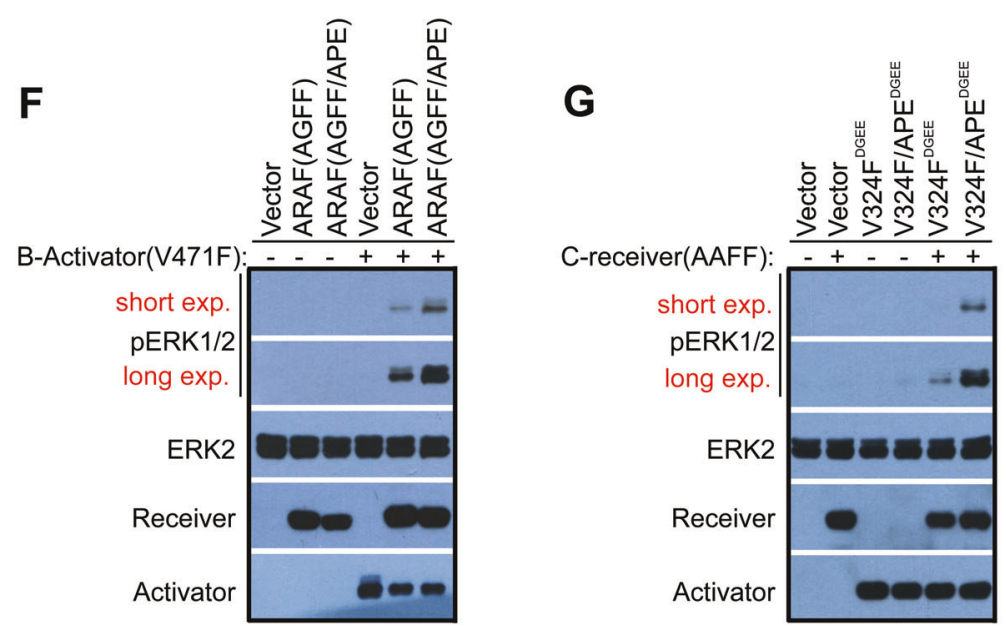

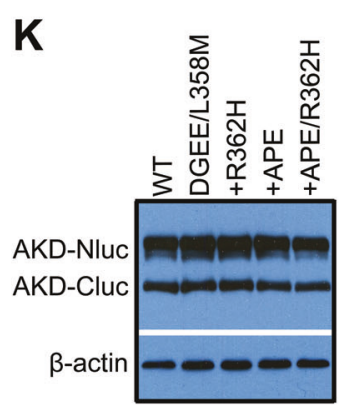

Fig. 3 The non-canonical APE motif decreases the activity of ARAF. a R-spine fusion together with acidic NtA motif fully activates ARAF. b The constitutively-active R-spine mutant of ARAF is resistant to the activation loop (AL) mutation but not to the central RH alteration in dimer interface. c ARAF has a non-canonical APE motif. The conserved APE motif is altered into AAE in ARAF, which might weaken the Glu-Arg salt bridge between APE motif and $\alpha \mathrm{H}-\alpha \mathrm{I}$ loop. d Schematic diagram showing the Glu-Arg slat bridge in CRAF. Schematic diagram of CRAF (PDB ID: 3OMV) was generated by using PyMOL software. $\mathrm{e}$ The conserved APE motif restores the activity of ARAF R-spine mutant with the central RH alteration in dimer interface. $\mathbf{f}-\mathbf{g}$ The conserved APE motif enhances both allosteric activator and receiver activities of ARAF. The RAF co-activation assays were carried out as in Fig. 2. $\mathbf{h}$ The alteration of APE motif makes BRAF Rspine mutant sensitive to the central RH alteration in dimer interface. $\mathbf{i}$ The alteration of APE motif or the breakage of Glu-Arg salt bridge makes CRAF R-spine mutant sensitive to the central RH alteration in dimer interface. $\mathbf{j}, \mathbf{k}$ The conserved APE motif enhances the dimer affinity of ARAF mutants. The dimer affinity of ARAF mutants is measured by complementary split luciferase as in Fig. 1a $(n=5$, *** $p$ $<0.001)$. In $\mathbf{a}, \mathbf{b}, \mathbf{e}$ and $\mathbf{h}, \mathbf{i}$ the activity of RAF mutants in 293T transfectants was measured by anti-phospho-ERK1/2 immunoblot. All images are representative of at least three independent experiments. "KD" represents for "kinase domain" in the full text 
APE motif forms a salt-bridge with Arg in the $\alpha \mathrm{H}-\alpha \mathrm{I}$ loop (Fig. 3d), which has been shown to play a critical role in the regulation of protein kinase A [42]. Since Ala is a helixfavored residue, we thought that a substitution of Pro with Ala would generate a longer $\alpha E F$ with a more rigid $\mathrm{N}$ terminus, and therefore weaken/break the Glu-Arg saltbridge and impair kinase function. To test this hypothesis, we mutated Ala back to Pro in the APE motif of ARAF (DGEE/L358M), and found that this mutant was resistant to the central RH alteration in dimer interface as well as the homologous BRAF and CRAF mutants described in our previous studies [20, 37] (Fig. 3e). Furthermore, ARAF activator and receiver with a canonical APE motif exhibited much stronger activity than their wild-type counterparts in co-activation assays (Fig. 3f, g). On the other hand, a substitution of Pro with Ala in the APE motif of BRAF and CRAF sensitized their constitutively-active R-spine mutants, BRAF(L505M) and CRAF(DDEE/L397M), to the central RH alteration in dimer interface (Fig. 3h, i). Moreover, a breakage of the Glu-Arg salt-bridge by replacing Arg with Gln also led to the sensitivity of CRAF (DDEE/ L397M) to the central RH alteration in dimer interface (Fig. 3i). Together, these data demonstrate that the different APE motifs of RAF isoforms are responsible for the differential activities observed between ARAF and the other RAF paralogs.

In order to further address how the non-canonical APE motif of ARAF dampens its functional activities, we next investigated whether it affects the dimer affinity of ARAF by using complementary split luciferase assay as described above. As shown in Fig. 3j, k, a canonical APE motif conferred the higher dimer affinity of ARAF R-spine mutants, comparing ARAF(DDEE/L358M) with ARAF (DDEE/L358M/APE), and ARAF(DDEE/L358M/R362H) with ARAF(DDEE/L358M/R362H/APE), which indicates that the non-canonical APE motif of ARAF decreases its dimer affinity. Since the dimerization plays a critical role in RAF activation, this data indicates that the lower dimer affinity arising from the non-canonical APE motif leads to the weaker activities of ARAF among RAF isoforms. In addition, the lower dimer affinity of ARAF(DDEE/L358M) and its sensitivity to the central $\mathrm{RH}$ alteration in dimer interface in contrast to ARAF(DDEE/L358M/APE), suggest that the active R-spine mutants of ARAF might function as a dimer to activate MEK-ERK pathway even if they do not require dimerization-driven transactivation for triggering their activity.

\section{Similar in-frame deletions of dimeric protein kinases exist in cancer genomes}

Dimerization-driven allostery plays a key role not only in the activation of RAF kinase but also in that of many other protein kinases $[43,44]$. Besides $\operatorname{ARAF}(\triangle \mathrm{QA})$, an oncogenic BRAF mutant with $\beta 3-\alpha C$ loop deletion $(\triangle N$ NTAP) has been reported although its activation mechanism remains controversial [31-34]. We here aimed to explore all kinase mutants with similar deletions in cancer genomes and to asses the importance of these mutations in human cancers. To this end, we interrogated the ICGC (International Cancer Genome Consortium) database, the cBioportal for Cancer Genomics database, and the COSMIC (Catalogue of Somatic Mutations in Cancer) database, and summarized all kinase mutants with similar in-frame deletions of $\beta 3-\alpha \mathrm{C}$ loop, including those reported in literatures [31-34], in Table S2. Among these mutants, the EGFR exon19 del is highly prevalent and has been shown to elevate kinase activity by promoting side-to-side homodimerization [45]. Other similar kinase mutants include those of BRAF, JAK1 and ERB B2, which (except JAK1 mutants, which have not been tested) have shown elevated kinase activity (see below and Figure S2).

\section{In-frame deletions of $\beta 3$-aC loop activate BRAF as well as CRAF by enforcing homo-dimerization}

To characterize BRAF mutants in Table S2 and determine whether they are activated by enhanced homodimerization, we expressed these mutants in $293 \mathrm{~T}$ cells and fibroblasts. All these mutants stimulated the MEK-ERK pathway independent of upstream stimuli or endogenous RAF molecules (Fig. 4a, b and S3A), indicating that they are constitutively active. However, these mutants exhibited differential resistance to the central RH alteration (R509H) in dimer interface (Fig. 4c). This alteration had no effect on the activity of $\operatorname{BRAF}(\triangle N$ VTAP $)$, partially inhibited that of $\operatorname{BRAF}(\triangle \mathrm{MLN})$, and completely blocked that of BRAF ( $\triangle$ NVTAPT). We reasoned that this discrepancy among BRAF mutants might arise from their different dimer affinity/stability. To test this hypothesis, we carried out coimmunoprecipitation assays, and found that indeed these mutants had enhanced but different propensities to form dimers, with $\Delta$ NVTAP $>\Delta \mathrm{MLN}>\Delta \mathrm{NVTAPT} \approx \Delta \mathrm{QA}>$ WT, independently of ERK1/2-mediated feedback [46] (Fig. 4d and S3B\&C). The R509H alteration prevented the homodimerization of $\operatorname{BRAF}(\triangle N V T A P T)$ and $\mathrm{BRAF}$ ( $\triangle \mathrm{QA})$, partially that of $\mathrm{BRAF}(\triangle \mathrm{MLN})$, and weakly that of $\operatorname{BRAF}(\triangle N V T A P)$. Previous studies have shown that although the central Arginine alteration impairs the dimerization-driven transactivation of wild-type RAFs [15], it makes up less than $20 \%$ dimer interface [11]. Therefore, the resistance of $\operatorname{BRAF}(\Delta \mathrm{MLN})$ and $\operatorname{BRAF}(\Delta \mathrm{NVTAP})$ to the central $\mathrm{R} 509 \mathrm{H}$ alteration in dimer interface does not exclude that these two BRAF mutants are activated through dimerization-driven transactivation by virtue of their much stronger dimer affinity than wild-type counterpart. To 

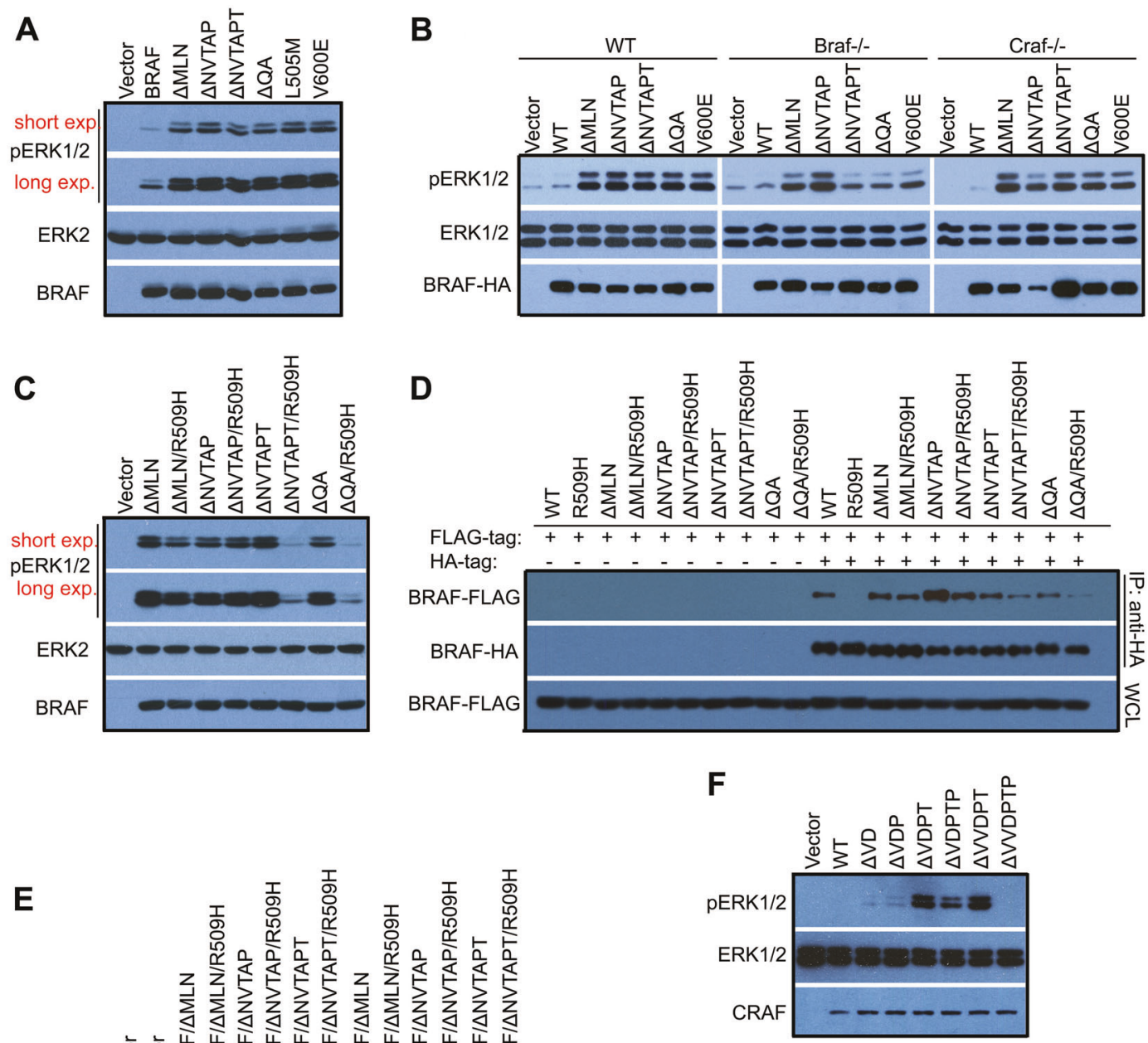

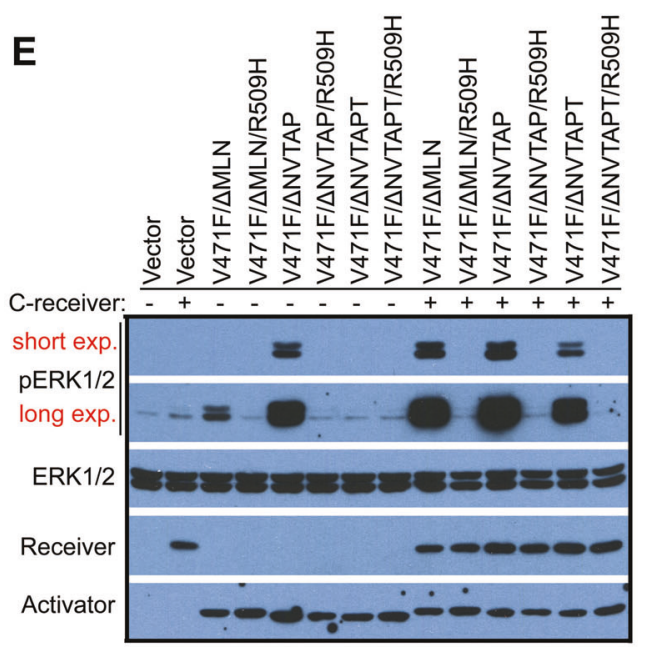

Fig. 4 In-frame $\beta 3-\alpha C$ loop deletions activate RAF kinase by enhancing homodimerization. a-c BRAF mutants with in-frame $\beta 3-\alpha \mathrm{C}$ loop deletions activate the MEK-ERK pathway independent of endogenous RAF molecules, and exhibit distinct resistance to the central R509H alteration in dimer interface. The activity of BRAF mutants in 293T transfectants or MEF stable cell lines was measured by anti-phosphoERK1/2 immunoblot. d BRAF mutants with in-frame $\beta 3-\alpha \mathrm{C}$ loop deletions have an elevated but differential dimer affinity/stability. BRAF mutants with FLAG- or HA-tag were coexpressed in $293 \mathrm{~T}$ cells, and immunoprecipitated by anti-HA beads and detected by

further demonstrate that these BRAF mutants, especially $\operatorname{BRAF}(\triangle N V T A P)$ and $\operatorname{BRAF}(\triangle M L N)$, are activated by enhanced homodimerization, we performed the RAF coactivation assay $[20,36,37,40,41]$ by using their kinase- dead V471F mutants as allosteric activators. All activators tested in this study strongly stimulated the catalytic activity of CRAF receivers (Fig. 4e), and particularly activators derived from $\operatorname{BRAF}(\triangle \mathrm{NVTAP})$ and $\mathrm{BRAF}(\triangle \mathrm{MLN})$ could 
even trigger endogenous RAF molecules (Fig. 4e lane5 and lane3). The central $\mathrm{R} 509 \mathrm{H}$ alteration in dimer interface was unable to prevent these two strong allosteric activators from triggering BRAF receivers (Figure S3D). Since the noncanonical APE motif had been also shown to decrease the dimer affinity in RAF molecules, we next introduced it together with the central R509H alteration into BRAF ( $\triangle$ NVTAP) mutant, and found that this combined alteration completely blocked the activity of $\operatorname{BRAF}(\Delta N \mathrm{NTAP})$ (Figure S3E). Taken together, our data demonstrates that all BRAF mutants with in-frame deletions of $\beta 3-\alpha \mathrm{C}$ loop are activated by enhanced homodimerization.

Unlike ARAF and BRAF, we did not find any CRAF mutants with in-frame deletions of $\beta 3-\alpha C$ loop in databases. To test whether CRAF can be activated by this type of mutations, we constructed mutants homologous to those of ARAF and BRAF. When expressed in 293T cells, $\Delta$ VDPT, $\triangle$ VDPTP, and $\triangle$ VVDPT mutants of CRAF, but not other mutants, strongly activated the MEK-ERK pathway independent of upstream stimuli (Fig. $4 \mathrm{f}$ and S3F\&G), and exhibited differential resistance to the central $\mathrm{RH}$ alteration $(\mathrm{R} 401 \mathrm{H})$ in dimer interface (Fig. 4g). Since we had showed that BRAF was activated by mutations $(\triangle \mathrm{QA}$ and $\Delta$ QL) homologous to $\operatorname{ARAF}(\Delta Q A)$, we hence determined whether ARAF could be triggered by mutations homologous to BRAF( $\triangle \mathrm{MLN}, \triangle \mathrm{NVTAP}$, and $\triangle$ NVTAPT). However, none of these alterations activated ARAF (Figure S3H).

\section{All BRAF mutants with variable deletions of $\beta 3-\alpha C$ loop have a strong transforming potential, and a robust resistance to Vemurafenib but not LY3009120 that correlates with their dimer affinity}

Although the oncogenic potential and resistance to RAF inhibitor of $\mathrm{BRAF}(\triangle \mathrm{NVTAP})$ has been reported recently [31, 34], whether all BRAF mutants with in-frame deletions of $\beta 3-\alpha C$ loop are able to function as cancer drivers and their pharmacological characteristics are not clear. To address these questions, we first measured the oncogenic potential of these mutants by foci formation assays. As shown in Fig. 5a and S4A\&B, all BRAF mutants with inframe deletions of $\beta 3-\alpha C$ loop transformed immortalized fibroblasts and induced foci formation independent of endogenous RAF molecules, suggesting that they can function as drivers to induce cancers. Further, we examined their sensitivities to the RAF inhibitor Vemurafenib, and found that all mutants exhibited a robust resistance to this drug, ranking as $\operatorname{BRAF}(\triangle N V T A P)>\operatorname{BRAF}(\Delta M L N)>$ $\operatorname{BRAF}(\Delta \mathrm{NVTAPT}) \approx \mathrm{BRAF}(\Delta \mathrm{QA}) » \mathrm{BRAF}(\mathrm{V} 600 \mathrm{E}) \quad$ (Fig. $5 b, c)$, which correlates with their dimer affinity/stability. However, all these mutants had similar sensitivities to the RAF dimer inhibitor, LY3009120, which are comparable with that of BRAF(V600E) in A101D melanoma cell line (Fig. 5d, e).

\section{The high dimer affinity of kinase-dead BRAF mutants with $\beta 3-\alpha C$ loop deletions bypasses the requirement of active RAS to drive tumorigenesis}

The inhibitor-loading has been shown to fuse the catalytic spine of RAF molecules, which can be mimicked by the Val to Phe mutation in ATP-binding pocket [20, 36-41]. Compound BRAF mutants with both $\beta 3-\alpha \mathrm{C}$ loop deletion and Val471Phe, especially $\operatorname{BRAF}(\Delta \mathrm{NVTAP} / \mathrm{V} 471 \mathrm{~F})$, lacked catalytic activity but were able to activate the MEK-ERK pathway through triggering wild-type RAFs (Fig 4e). The kinase-dead BRAF( $\triangle$ NVTAP/V471F)induced foci formation in vitro and tumor formation in vivo even in the absence of active RAS, but dependent on endogenous RAF molecules (Fig. 5f-i and S4C). Since a previous study had shown that kinase-dead RAFs cooperate with active RAS to induce tumorigenesis [12], this data suggests that the high dimer affinity of RAF mutants could bypass the requirement of active RAS to driven cancer development.

\section{Active RAF kinases function as a dimer to phosphorylate MEK}

The activation of the RAF/MEK/ERK kinase cascade is a very complex process and its underlying molecular basis is not completely understood [45-49]. In current model, RAF and MEK form a face-to-face dimer in quiescent cells. Upon stimulation, two RAF-MEK dimers are brought together and assemble a transient tetramer through back-toback RAF dimerization, which not only activates RAF but also facilitates subsequent MEK activation [19]. However, how active RAF activates MEK is not clear. To elucidate this process, we first tested whether the dimerization of active RAF is required for MEK activation by using BRAF mutants with in-frame deletions of $\beta 3-\alpha C$ loop since these mutants have different dimer affinity/stability. As shown in Fig. 6a, those constitutively-active BRAF mutants with low dimer affinity/stability such as $\operatorname{BRAF}(\Delta \mathrm{NVTAPT})$ and $\operatorname{BRAF}(\triangle \mathrm{QA})$ lost their catalytic activity towards MEK in vitro when purified by immunoprecipitation, in contrast to those mutants with high dimer affinity/stability which retained catalytic activity toward MEK. We reasoned that the loss of activity of BRAF mutants in vitro arises from their dimer dissociation during purification. To test this hypothesis, we strengthened the dimers of BRAF $(\triangle \mathrm{NVTAPT})$ and $\mathrm{BRAF}(\triangle \mathrm{QA})$, respectively, by GST fusions [50], and found that it restored their catalytic activity in vitro (Fig. 6b). This phenomenon was also seen with a homologous ARAF mutant $(\triangle \mathrm{QA})$ whose in vitro 


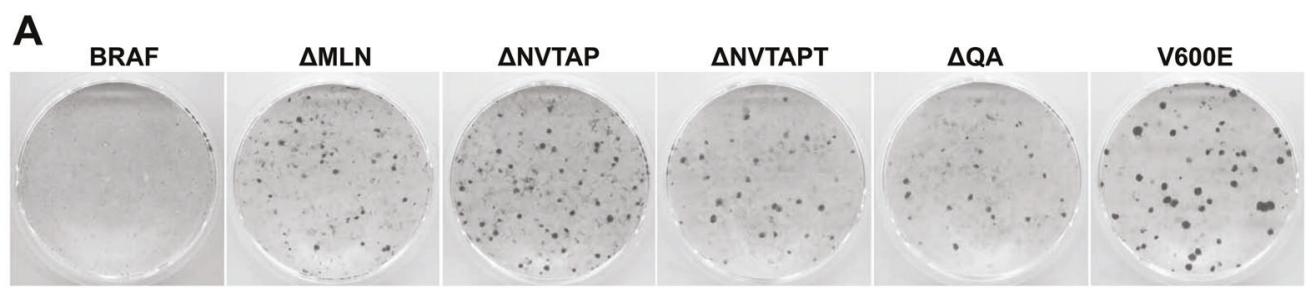

B

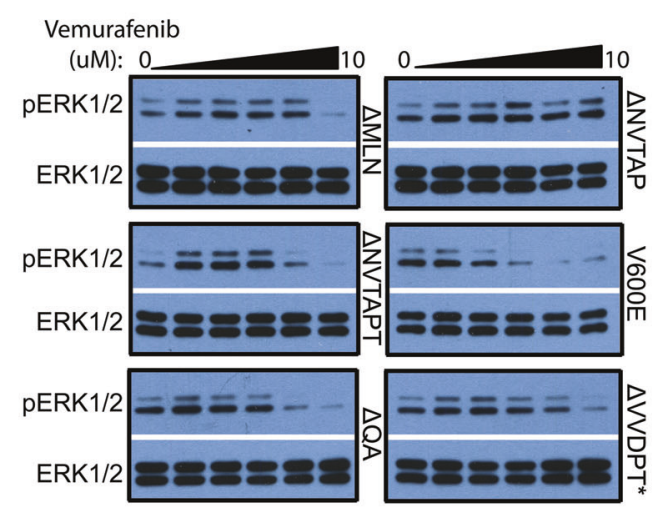

D LY3009120

(uM): 0 pERK1/2

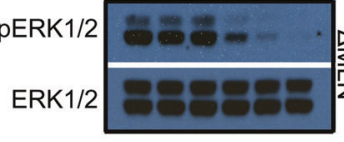

pERK1/2

ERK1/2
C

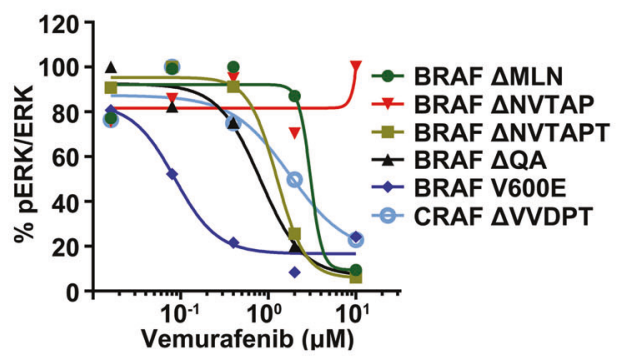

E

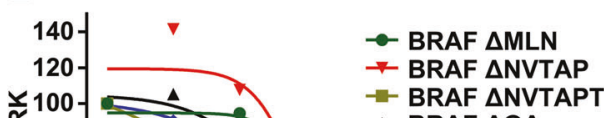

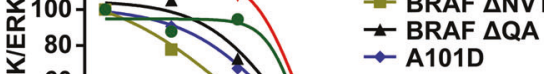

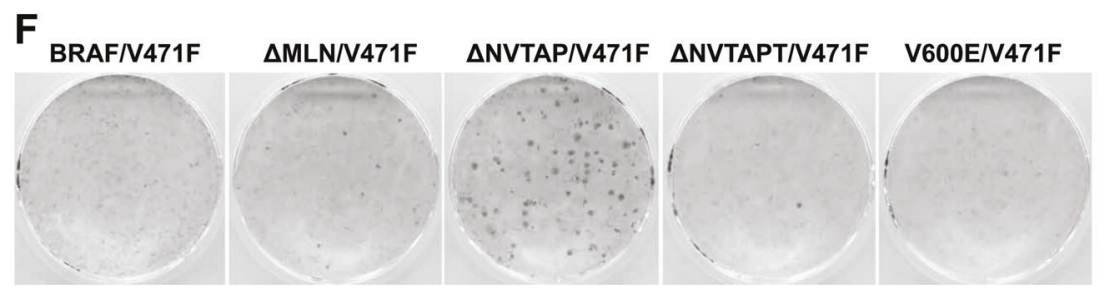

G

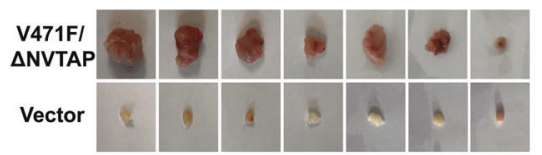

\section{H}

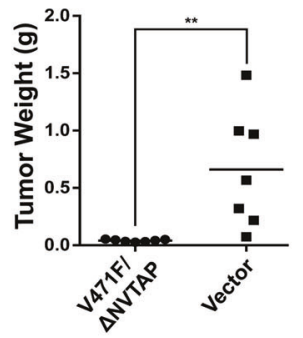

I

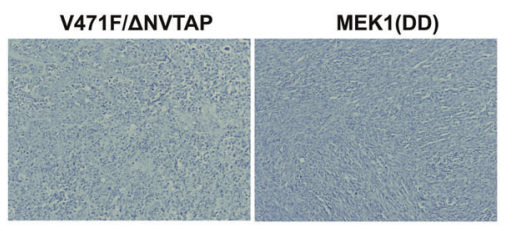

catalytic activity was rescued by GST fusion (Fig. 6c). As reported before [20, 51] and shown in this study, both alterations of the central RH in dimer interface and the APE motif significantly impair but do not completely abolish the dimer formation of RAF molecules. We therefore next examined the effect of these alterations on the in vitro catalytic activity of active RAF mutants. Among three active ARAF R-spine mutants, only the one with a high 
Fig. 5 BRAF mutants with in-frame $\beta 3-\alpha C$ loop deletions have a strong transforming ability and a robust but differential resistance to Vemarufenib. a BRAF mutants with in-frame $\beta 3-\alpha C$ loop deletions induce foci formation when stably expressed in immortalized fibroblasts. The foci formation assay was carried out as in Fig. 1c. b, c BRAF mutants with in-frame $\beta 3-\alpha C$ loop deletions exhibit a robust but differential inhibitor resistance. Stable fibroblast cells that express individual BRAF mutants with in-frame $\beta 3-\alpha \mathrm{C}$ loop deletions were treated with Vemurafenib for $4 \mathrm{~h}$, and phospho-ERK1/2 was probed by immunoblot and quantified by using Image J. The graphs were generated by using GraphPad Prism 6. d,e BRAF mutants with in-frame $\beta 3-\alpha C$ loop deletions have approximate sensitivities to RAF dimer inhibitor, LY3009120. The drug sensitivities of BRAF mutants were measured as in b, c. The BRAF(V600E)-harboring melanoma cell line, A101D, was used as control. $\mathbf{f}-\mathbf{i}$ Catalytic spine-fused BRAF mutants with in-frame $\beta 3-\alpha C$ loop deletions have variable oncogenic potentials in vitro and in vivo. $\mathbf{f}$ The oncogenic potential of BRAF mutants was measured by the foci formation assay as in $\mathbf{a}$. $\mathbf{g}$ Xenograft tumors were generated in NOD/SCID mice from immortalized fibroblasts that express BRAF mutants as described in Materials and Methods section. $\mathbf{h}$ The weight of xenogrfat tumors from $\mathrm{E}\left(n=7\right.$ for each group, ${ }^{*} p<<$ 0.01). i Representative images from histological section staining of xenograft tumors from $\mathrm{E}(n=7)$. The MEK1DD-driven xenograft tumor served as control. All images are representative of at least three independent experiments dimer affinity (see Fig. 3j, k) maintained its catalytic activity in vitro after purification (Fig. 6d), and GST fusion restored that of the other two mutants with a low dimer affinity (Fig. 6e). Similar to that of ARAF mutants, active CRAF mutants with an alteration of RH, or of APE, lost their catalytic activity in vitro, which was recovered by GST fusion-enhanced dimerization (Fig. 6f). As to active BRAF R-spine mutants, the alterations of R509H, or APE, inhibited their catalytic activity in vitro by different extends, which was also relieved by the GST fusion approach (Fig. $6 \mathrm{~g}$ ). The loss of in vitro catalytic activity of RAF mutants with low dimer affinity could be also rescued by other dimeric molecular fusions (data not shown), or partially restored with a gentle wash of PBS during purification (Figure S5), which excludes the potential artificial effect arising from GST fusion. Together, these data indicate that all RAF isoforms would function as dimers to phosphorylate MEK.

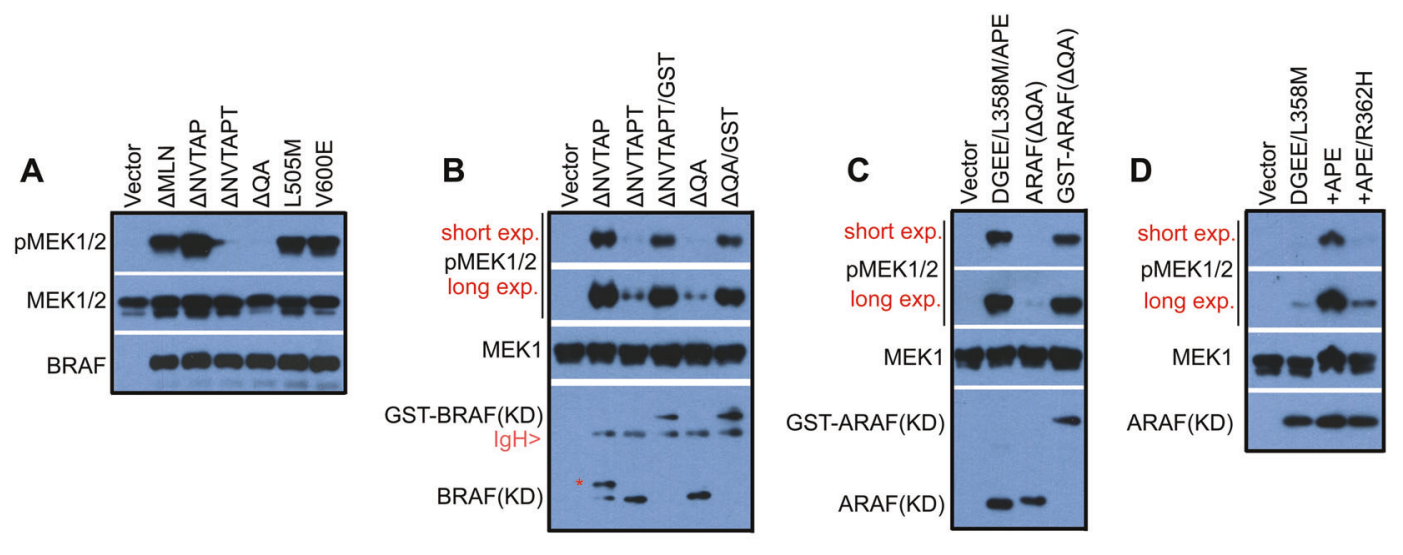

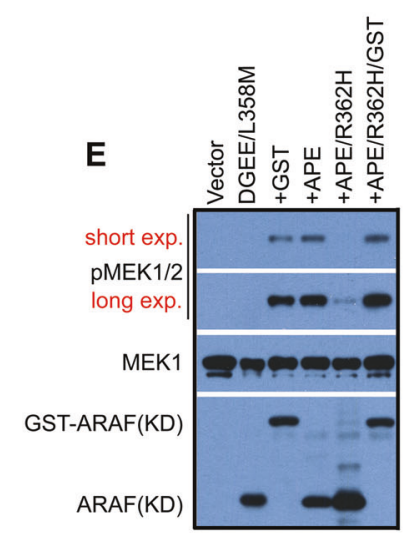

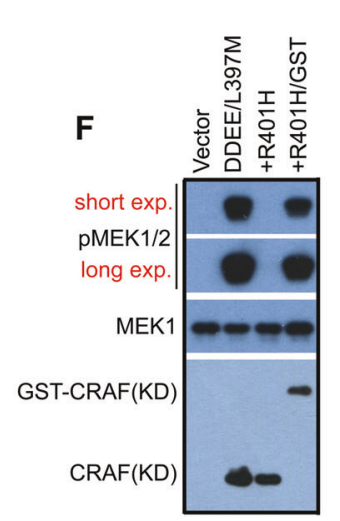

fusion restores in vitro the kinase activity of constitutively active Rspine mutants of ARAF, CRAF, and BRAF with low dimer affinity. In a-g, all RAF mutants were expressed in $293 \mathrm{~T}$ cells and purified by immunoprecipitation, and their activity was measured by in vitro kinase assays as described before [20, 37]. All images are representative of at least three independent experiments

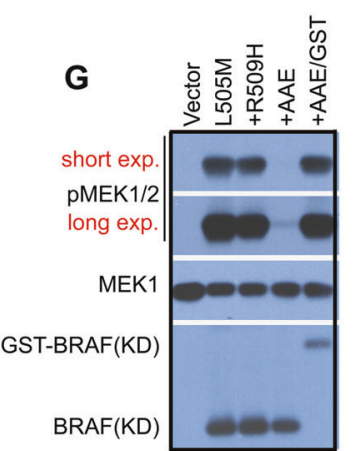

Fig. 6 Active RAF mutants phosphorylate MEK in a dimer-dependent manner. a,b BRAF mutants with in-frame $\beta 3-\alpha C$ loop deletions lose their catalytic activity in vitro upon purification if they have a low dimer affinity, which is rescued by GST fusion. The band labeled with "*” in lane 2 of B represents the highly phosphorylated BRAF(KD, $\triangle N V T A P T)$. c Like $\operatorname{BRAF}(\triangle Q A)$, purified $\operatorname{ARAF}(\triangle Q A)$ loses its kinase activity in vitro, which is rescued by GST fusion. d-g GST
SPRINGER NATURE 


\section{BRAF(V600E) also phosphorylates MEK through a dimer-dependent manner}

Given its resistance to the central $\mathrm{RH}$ alteration in dimer interface and sensitivity to Vemurafenib, BRAF(V600E), a dominant mutant in RAF mutation spectrum, has been proposed to function as a monomer to activate MEK [26]. However, recent studies showed that BRAF(V600E) exists as dimers or high-order oligomers in cells [51-53]. This prompted us to determine whether, unlike other active RAF mutants, BRAF(V600E) truly phosphorylates MEK in a dimer-independent manner. Since BRAF(V600E) has an enhanced propensity to form dimers [51-53], the central RH alteration $(\mathrm{R} 509 \mathrm{H})$ in dimer interface is unable to dissociate its dimers completely. Hence, our approach was to replace one protomer in the dimer of $\mathrm{BRAF}(\mathrm{V} 600 \mathrm{E})$ with one dysfunctional BRAF mutant (loss of both catalytic and allosteric activities), and measured the catalytic activity of BRAF(V600E) in heterodimers. To generate such a dysfunctional BRAF mutant, we mutated the residues of the kinase-dead $\operatorname{BRAF}(\triangle \mathrm{NVTAP} / \mathrm{V} 471 \mathrm{~F})$ that mediate the heterodimerization of BRAF with MEK [19] (Fig. 7a). Since BRAF utilizes two different groups of residues to bind MEK and RAF [19], the introduction of these mutations would not influence RAF dimerization. Unlike its prototype, the mutant, $\mathrm{BRAF}(\Delta \mathrm{NVTAP} / \mathrm{V} 471 \mathrm{~F} / \mathrm{R} 462 \mathrm{E} /$ I617R/F667A), heretofore referred to as $\mathrm{BRAF}(\Delta \mathrm{NVTAP} /$ $\mathrm{V} 471 \mathrm{~F})^{*}$, had no allosteric ability to trigger endogenous RAF molecules when expressed in 293T cells (Fig. 7b). Moreover, BRAF(V600E) bound to BRAF( $\mathrm{NVTAP} /$ $\mathrm{V} 471 \mathrm{~F})^{*}$ had little catalytic activity in vitro, in contrast to that bound to $\operatorname{BRAF}(\Delta \mathrm{NVTAP} / \mathrm{V} 471 \mathrm{~F})$ when purified from 293T co-transfectants (Fig. 7c). This suggests that a BRAF (V600E) molecule needs a partner able to hold MEK in order to phosphorylate it. To further confirm this finding, we introduced $\operatorname{BRAF}(\triangle \mathrm{NVTAP} / \mathrm{V} 471 \mathrm{~F}) *$ into $\mathrm{BRAF}$ (V600E)-dependent melanoma cell lines by lentiviral transductions and found that its expression downregulated phospho-ERK1/2 and inhibited cell growth in vitro and xenograft tumor growth in vivo (Fig. 7d-h).

As shown above, the APE motif of RAF kinases regulates their dimerization, likely through affecting the GluArg salt bridge between the APE motif and the $\alpha \mathrm{H}-\alpha \mathrm{I}$ loop, and the alteration of APE motif into non-canonical AAE dramatically decreases RAF dimer affinity/stability. Therefore, we next determined whether an alteration of APE motif into AAE would impair the catalytic activity of BRAF (V600E) in vitro, as which occurs in ARAF R-spine mutants. As shown in Fig. 7i, j and S6A, although the AAE variant of BRAF(V600E) had comparable ability with its prototype to phosphorylate MEK and thus turn on ERK signaling when expressed in $293 \mathrm{~T}$ cells, it lost its catalytic activity upon purification by immunoprecipitation, which was restored by GST fusion. Moreover, the combined alterations of AAE and R509H completely blocked the activity of BRAF(V600E) even in vivo, which was also recovered by GST fusion (Fig. 7i). To directly confirm that the discrepant catalytic activity among BRAF(V600E), BRAF(V600E/AAE), and BRAF(V600E/AAE/R509H), arises from their different dimer affinity/stability, we completed a co-immonprecipitation assay with a gentle wash of PBS as in Figure S5, and found that these BRAF variants had quite different ability to form dimers as BRAF (V600E) » BRAF(V600E/AAE) » BRAF(V600E/AAE/

R509H) (Figure S6B). Altogether, these data demonstrate that like other RAF molecules, BRAF(V600E) also functions as a dimer to activate MEK.

\section{Discussion}

The dimerization of RAF kinase not only plays a critical role in the activation of the RAF/MEK/ERK kinase cascade [9-17], but also contributes to drug resistance in cancer therapy $[11,12,14,26]$. Previous studies have shown that the RAF dimerization could be improved by active RAS, inhibitor binding, gene fusions or alternative splicing [9, $11-14,17,22-26,54]$. However, whether it can be achieved by other ways remains unknown. Recently, some oncogenic RAF mutants with $\beta 3-\alpha \mathrm{C}$ loop deletions have been reported by several groups [31-34], and both dimerdependent and -independent models have been suggested to explain how this type of mutations activates RAF [32, 34]. Chen et al. characterized $\mathrm{BRAF}(\triangle \mathrm{LNVTAP})$ that resembles $\operatorname{BRAF}(\triangle N V T A P T)$ in cancer genomes, and found that its activity was blocked by the central $\mathrm{R} 509 \mathrm{H}$ alteration in dimer interface [32]. On the other hand, Foster et al. showed that the other cancer-related mutant, $\operatorname{BRAF}(\triangle \mathrm{NVTAP})$ was resistant to the same alteration [34]. Both groups failed to understand the discrepancy among these RAF mutants. In this study, we systemically characterize all RAF mutants with $\beta 3-\alpha C$ loop deletions, and provide solid evidence that this type of mutations activates RAFs through improving homodimerization, which clarifies the controversial between those two groups.

Among RAF isoforms, BRAF and CRAF have been shown to function as both catalysis-competitive kinases and allosteric activators [21], while ARAF has a bare activity and been thought as a scaffold $[55,56]$. By characterizing the ARAF mutant with a $\beta 3-\alpha \mathrm{C}$ loop deletion $(\Delta \mathrm{QA})$, we demonstrate that like the other two paralogs, ARAF has both catalytic and allosteric activities though less. Further, we elucidate that the weak activity of ARAF arises from its non-canonical APE motif that decreases its dimer propensity likely by weakening the Glu-Arg salt bridge between the APE motif and the $\alpha \mathrm{I}-\alpha \mathrm{H}$ loop. Our data clearly 


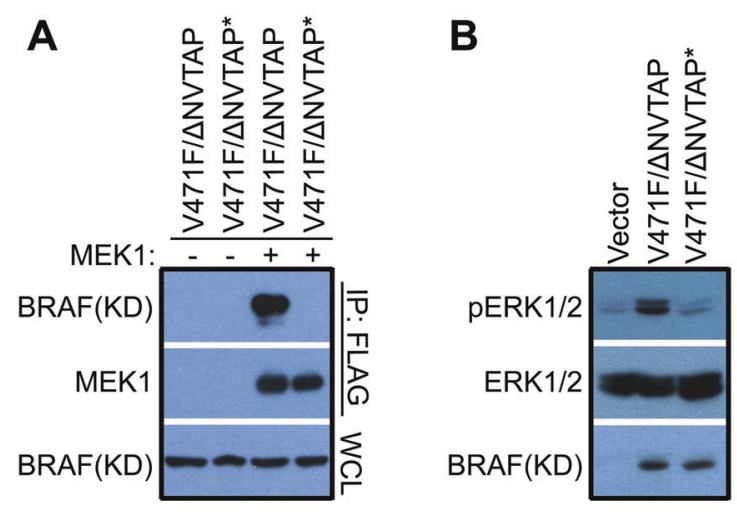

C (V471F/ANVTAP)-HA: -

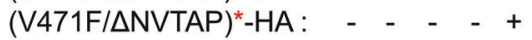

(V600E)-FLAG:
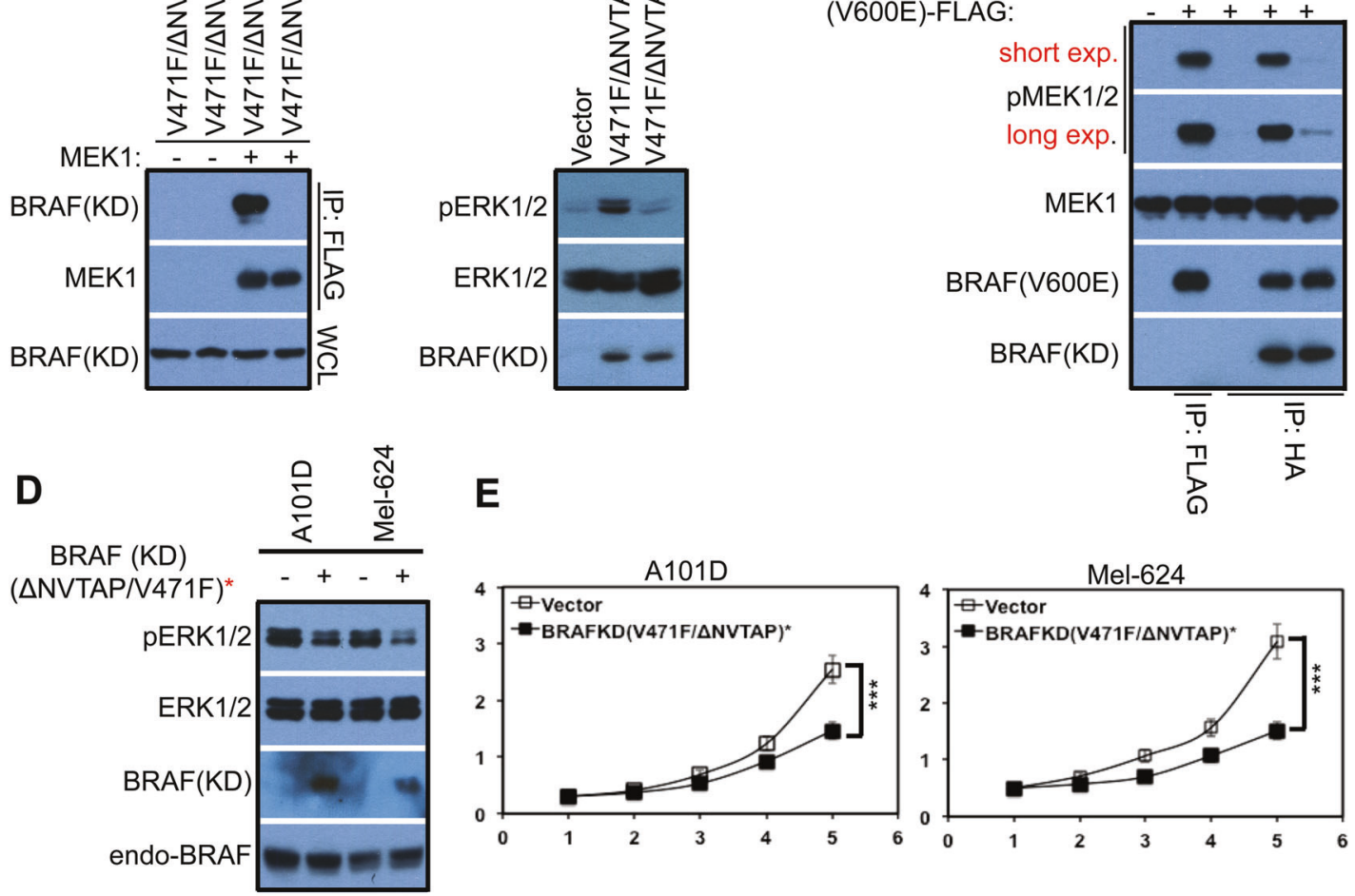

E

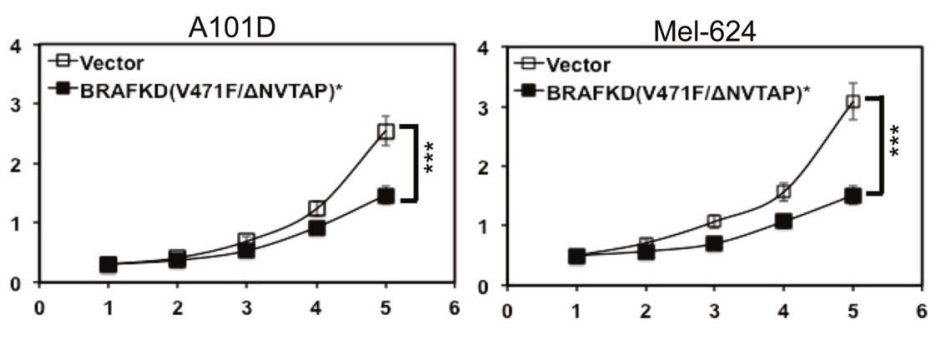

$\mathbf{F}$

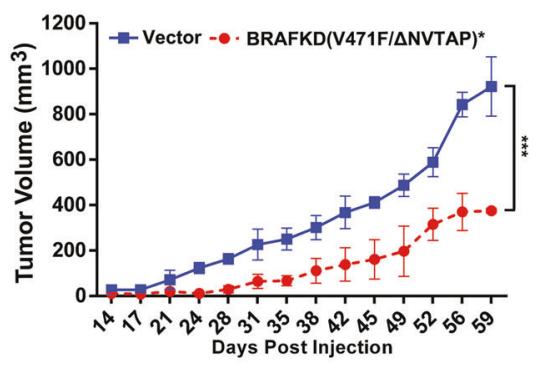

H

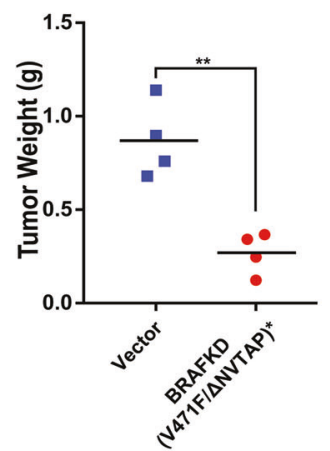

I

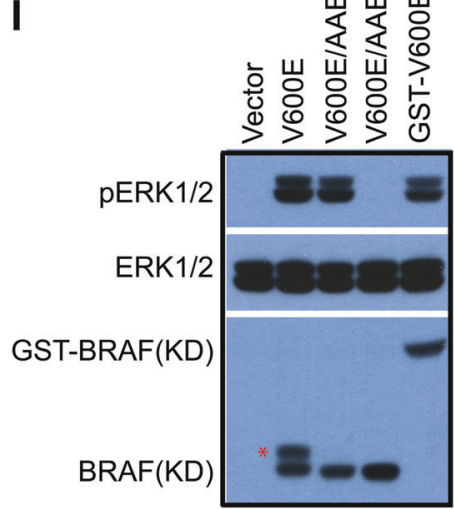

G
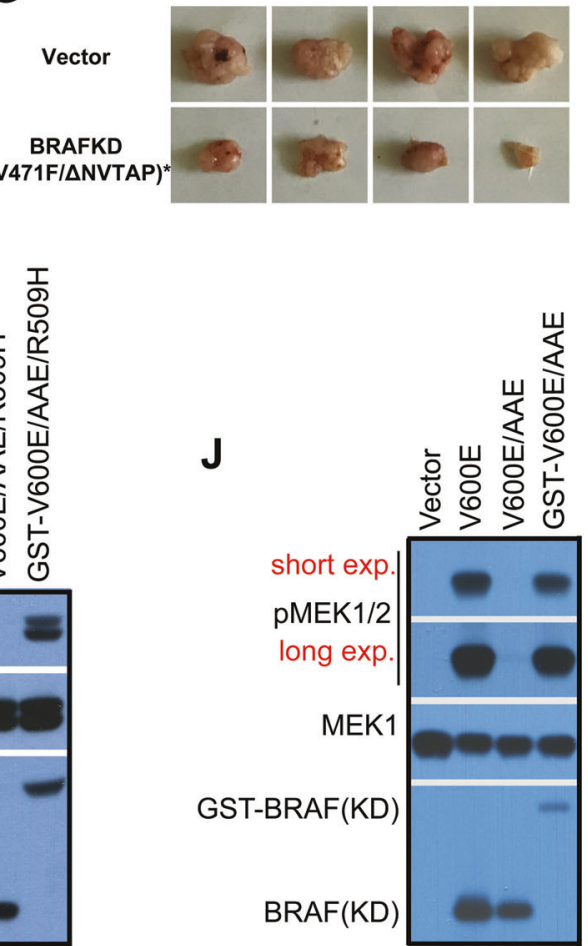

explain why ARAF has less ability to stimulate downstream MEK-ERK signaling.

In-frame deletions of $\beta 3-\alpha C$ loop activate not only RAFs but also other dimeric protein kinases by enhancing homodimerization. Remarkably, we found this type of mutation in BRAF, ERBB2, JAK2, and EGFR in cancer genomes, suggesting that the dimerization works as a principal mechanism to regulate the activity of these 
Fig. 7 BRAF(V600E) functions as a dimer to activate MEK. a, b The kinase-dead BRAF mutant with high RAF dimer affinity requires ability to heterodimerize with MEK1 for transactivating wild-type RAF molecules. a BRAF( $\Delta$ NVTAPT/V471F/R462E/I617R/ F667A), referred to as $\mathrm{BRAF}(\triangle \mathrm{NVTAPT} / \mathrm{V} 471 \mathrm{~F}) *$ below, was generated by PCR and its ability to heterodimerize with MEK1 was measured by coimmunoprecipitation as in Fig. 1e. b Unlike its prototype, BRAF $(\triangle \mathrm{NVTAPT} / \mathrm{V} 471 \mathrm{~F}) *$ is not able to activate endogenous RAF molecules when expressed in $293 \mathrm{~T}$ cells. BRAF mutants were expressed in $293 \mathrm{~T}$ cells, and their activity was measured by anti-phospho-ERK1/2 immunoblot. c BRAF(V600E) loses its catalytic activity once dimerizing with $\mathrm{BRAF}(\triangle \mathrm{NVTAPT} / \mathrm{V} 471 \mathrm{~F}) *$. BRAF(V600E) that binds to $\operatorname{BRAF}(\Delta \mathrm{NVTAPT} / \mathrm{V} 471 \mathrm{~F}) *$ was purified by immunoprecipitation from 293T cotransfectants and its activity was measured by in vitro kinase assay as in Fig. 6. BRAF(V600E) that binds to BRAF $(\triangle \mathrm{NVTAPT} / \mathrm{V} 471 \mathrm{~F})$ was expressed and purified from $293 \mathrm{~T}$ cotransfectants, and serviced as a control. $\mathbf{d}-\mathbf{h}$ The stable expression of BRAF $(\triangle \mathrm{NVTAPT} / \mathrm{V} 471 \mathrm{~F}) *$ in $\mathrm{BRAF}(\mathrm{V} 600 \mathrm{E})$-dependent melanoma cell lines dampens MEK-ERK signaling, and inhibits cell growth in vitro and xenograft tumor growth in vivo. d-e, BRAF( $\triangle$ NVTAPT/V471F)* was stably expressed in A101D and Mel-624 cell lines, and phosphoERK1/2 and cell growth were measured respectively by immunoblot or by cell counting $(n=5, * * * p<0.001)$. f-h The growth curve, the photos and the weight of xenograft tumors derived from A101D melanoma cell lines that stably express $\operatorname{BRAF}(\Delta \mathrm{NVTAPT} / \mathrm{V} 471 \mathrm{~F}) *$ or empty vector $\left(n=4\right.$ for each group, $\left.{ }^{* * *} p<0.001, * * p<0.01\right) . \mathbf{i}, \mathbf{j}$ The activity of BRAF(V600E) with non-canonic APE motif is abolished by the central RH alteration in dimer interface in vivo or upon purification in vitro by immunoprecipitation, which is restored by GST fusion. $\mathbf{i}$ The APE alteration of BRAF(V600E) has little effect on its activity in cells, but makes BRAF(V600E) sensitive to the central RH mutation in dimer interface. BRAF mutants were expressed in 293T cells and their activity was measured by anti-phospho-ERK1/2 immunoblot. * In lane 2 indicates a post-translational modified version of BRAF(V600E). $\mathbf{j}$ The BRAF(V600E) with non-canonic APE loses its catalytic activity upon immunoprecipitation, which can be rescued by GST fusion. BRAF mutants were expressed in $293 \mathrm{~T}$ cells and purified by immunoprecipitation, and their activity was measured by in vitro kinase assay as in Fig. 6. All images are representative of at least three independent experiments

kinases. All BRAF mutants with variable deletions of $\beta 3$ $\alpha \mathrm{C}$ loop exhibit the elevated albeit differential dimer affinity, which is reflected by their different resistance to the central $\mathrm{R} 509 \mathrm{H}$ alteration in dimer interface. The strong dimer affinity of $\mathrm{BRAF}(\triangle \mathrm{NVTAP})$ bypasses the requirement of active RAS for its kinase-dead version to activate endogenous RAF and transform cells, suggesting that the dimerization of RAF is a dynamic equilibrium in cells and active RAS shifts this equilibrium in the favor of dimers.

A strong dimerization is required for transactivation of RAF molecules, whereas a weak dimerization is still indispensable for their catalytic function. The previous conclusion that active RAF kinase may function as a monomeric enzyme is mainly based on the data from the central RH alteration in dimer interface of RAF molecules. Recent studies revealed that this alteration was not able to completely dissociate RAF dimers although it blocked the dimerization-driven transactivation of RAF molecules $[20,51]$. Here, we found that this mutation does not block the dimerization-driven transactivation RAF mutants with high dimer affinity, such as BRAF $(\triangle N V T A P)$ and $B R A F(\triangle M L N)$, which calls for a reevaluation of previous data resulting from the central $\mathrm{RH}$ alteration in dimer interface. Constitutively active RAF mutants including BRAF(V600E) activate MEK in a dimer-dependent manner even if they do not require a dimerization to trigger their activity, implying that RAF kinase serves as both an enzyme and a MEK-docking platform in its catalysis process. This is further supported by that the kinase-dead BRAF mutant with high dimer affinity is not able to activate downstream MEK-ERK pathway through wild-type RAF molecules if it cannot bind MEK (Fig 7a, b). These findings suggest that the MEK-docking platform function of RAF kinase can be used as a target to develop novel therapeutic inhibitors against both RAS- and RAF-driven cancers. In current cancer therapies, the efficacy of RAF and MEK inhibitors has been severely limited by intrinsic and acquired resistances arising from the paradoxical activation or reactivation of RAF/MEK/ERK kinase cascade [57]. Such a new class of docking inhibitor would cover all type of cancers driven by hyperactive RAS/RAF/MEK/ERK signaling, and thus potentially have a greater and longer efficacy. Our study sheds a light on the treatment of RAS/RAF-driven cancers and has important clinic implications.

\section{Materials and methods}

\section{Biochemicals}

Antibodies used in this study included: anti-phosphoERK1/ 2 (\#4370), anti-phosphoMEK1/2 (\#9154), and anti-MEK1/2 (\#9124) (Cell Signaling Technology); anti-BRAF (SAB5300503), anti-CRAF (SAB5300393), anti-FLAG (F3165) and anti- $\beta$-actin (A2228) (Sigma); anti-HA (MAB6875, Novus Biologicals); anti-ERK2 (sc-154, Santa Cruz Biotechnology); anti-ERK1/2 (A0229, AB clonal); anti-Ki67 (ab16667, Abcam); and HRP-labeled secondary antibodies (Jackson Laboratories). Vemurafenib and LY3009120 were purchased from Medchemexpress; and Dluciferin from Gold Biotechnology. All other chemicals were obtained from Sigma.

\section{Plasmids and cell lines}

cDNAs encoding proteins in this study were purchased from Addgene or synthesized by Integrated DNA Technologies. All mutations were generated by PCR and tagged with either FLAG or HA or His, and cloned into vectors by Gibson assembly. pCDNA3.1(+) vector (Invitrogen) was used for 
transient expression; viral vectors (Clontech) for stable expression; and pET-28a (Novagen) for bacterial expression.

Wild-type, $\mathrm{BRAF}^{-1-}$ and $\mathrm{CRAF}^{-1-} \mathrm{MEFs}$ were generated in previous study [58, 59]. Melanoma cell lines: MeWo, A101D, Mel-624 were obtained from ATCC.

\section{Protein expression and purification}

6xhis-tagged MEK1 (K97A) and 6xhis-tagged ERK2 (K52A) were expressed in BL21(DE3) strains and purified by using Nickel column (Qiagen) and following our previous protocol [60].

\section{Cell culture, transfection, and transduction}

All cell lines were maintained in DMEM medium with $10 \%$ FBS (Hyclone). Cell transfection were carried out by using the Biotool transfection reagent and following the manufacturer's protocol. To generate stable cell lines that express RAF or MEK1 mutants, viruses were prepared and applied to infect target cells according to our previous studies [20, $36,37,60,61]$. Infected cells were sorted by FACS or selected by using antibiotics.

\section{Immunoprecipitation, in vitro kinase assay, and Western blotting analysis}

Immunoprecipitations were performed as described previously [20, 36, 37]. Briefly, whole-cell lysates were mixed with either anti-HA (E6779), or anti-FLAG beads (A2220) (Sigma), rotated in cold room for $60 \mathrm{~min}$, and washed three times with RIPA buffer. For in vitro kinase assays, the immunoprecipitants were washed once with kinase reaction buffer ( $25 \mathrm{mM}$ HEPES, $10 \mathrm{mM} \mathrm{MgCl}, 0.5 \mathrm{mM} \mathrm{Na}_{3} \mathrm{VO}_{4}$, $0.5 \mathrm{mM}$ DTT, pH 7.4), then incubated with $20 \mu$ kinase reaction mixture ( 2 ug substrate and $100 \mathrm{mM}$ ATP in $20 \mu \mathrm{l}$ kinase reaction buffer) per sample at room temperature for $10 \mathrm{~min}$. Kinase reaction was stopped by adding $5 \mu \mathrm{l}$ per sample 5XLaemmli sample buffer. Immunoblotting was carried out as described before [20, 36, 37, 60, 61].

\section{Foci formation assay}

Immortalized MEFs infected with retroviruses encoding target proteins were plated at $5 \times 10^{3}$ cells per $60 \mathrm{~mm}$ dish, and fed every other day. Twelve days later, cells were fixed with $2 \%$ formaldehyde and stained with Giemsa solution (Sigma).

\section{Complementary split luciferase assay}

A total of 293T transfectants that express different pairs of Nluc- and Cluc-fused RAF proteins were plated in 24-well Krystal black image plates at the the seeding density of $2 \times$
$10^{5}$ per well. Twentyfour hour later, D-luciferin $(0.2 \mathrm{mg} / \mathrm{ml})$ with or without Vemurafenib $(10 \mu \mathrm{M})$ was added to the culture, and the incubation was allowed for $30 \mathrm{~min}$ before the luciferase signals were measured by Promega GloMax ${ }^{\circledR}$. Multi Detection System.

\section{Animal studies}

For xenograft experiments, female NOD/SCID mice (6 8 weeks) were injected with $3 \times 10^{6}$ cells per mice in 1:1 matrigel (Corning). Tumor volumes were monitored by digital calipers twice a week and calculated using the formula: volume $=(\text { width })^{2} \times$ length $/ 2$. At the experiment endpoint, mice were killed and tumors were collected for ex vivo analysis and subsequent histology. All operations were approved by the Animal Ethics Committee of NCCS.

\section{Immunohistochemistry staining}

Tumors were fixed in $10 \%$ buffered formalin overnight and embedded according to standard procedures. Tumor sections were cut to 4 um thickness, mounted on glass slides, and air-dried at room temperature. After antigen retrieval, tumor sections were stained with antibodies and then with hematoxylin. Images of tumor sections were taken with a bright light microscope at $10 x$.

\section{Statistical analysis}

All statistical analysis in this study was performed using GraphPad InStat (GraphPad Software, CA, USA). Statistic significance was determined by two-tailed Student's $t$-test in animal studies and error bars represent s.d. to show variance between samples in each group, or by one-sample $t$-test in other experiments and error bars represent s.d. to show variance between independent experiments.

Acknowledgements We thank the laboratories of Dr. Hui, Dr. Sabapathy, Dr. Virshup, and Dr. Anand for their help in experimental technologies. We also thank Dr. Andrey Shaw and Dr. Susan Taylor for their assistances. This study is supported by NCCRF startup grant (NCCRF-SUG-JH), NCCRF bridging grant (NCCRF-YR2016-JULBG1), NMRC seeding grants (NCCSPG-YR2015-JUL-14 and NCCSPG-YR2016-JAN-17), and Duke-NUS Khoo Bridge Funding Award (Duke-NUS-KBrFA/2017/0003), Asia Fund Cancer Research (AFCR2017/2019-JH) and SHF Research Grant (SHF/FG692S/2016).

Author contributions $\mathrm{JY}$ and $\mathrm{JH}$ designed the study; JY and $\mathrm{JH}$ searched databases/literatures for kinase mutations in cancer genomes; MB prepared experimental materials; JY, WHN, YW, HX, JJY, and $\mathrm{JH}$ carried out molecular biology, biochemistry, and cell biology experiments; JY, PL, and SPG constructed mouse xenografts; JY, PL, and $\mathrm{AL}$ performed immunohistology analysis; PL, MW, and $\mathrm{JH}$ supervised all experiments and interpreted experimental data; JH wrote the manuscript; $\mathrm{MB}$ and MW revised manuscript; and all authors commented and approved the manuscript. 


\section{Compliance with ethical standards}

Conflict of interest The authors declare that they have no conflict of interest.

Open Access This article is licensed under a Creative Commons Attribution 4.0 International License, which permits use, sharing, adaptation, distribution and reproduction in any medium or format, as long as you give appropriate credit to the original author(s) and the source, provide a link to the Creative Commons license, and indicate if changes were made. The images or other third party material in this article are included in the article's Creative Commons license, unless indicated otherwise in a credit line to the material. If material is not included in the article's Creative Commons license and your intended use is not permitted by statutory regulation or exceeds the permitted use, you will need to obtain permission directly from the copyright holder. To view a copy of this license, visit http://creativecommons. org/licenses/by/4.0/

\section{References}

1. Baccarini M. Second nature: biological functions of the Raf-1 "kinase". FEBS Lett. 2005;579:3271-7.

2. Wellbrock C, Karasarides M, Marais R. The RAF proteins take centre stage. Nat Rev Mol Cell Biol. 2004;5:875-85.

3. Dhillon AS, Hagan S, Rath O, Kolch W. MAP kinase signalling pathways in cancer. Oncogene. 2007;26:3279-90.

4. Roberts PJ, Der CJ. Targeting the Raf-MEK-ERK mitogenactivated protein kinase cascade for the treatment of cancer. Oncogene. 2007;26:3291-310.

5. Schubbert S, Shannon K, Bollag G. Hyperactive Ras in developmental disorders and cancer. Nat Rev Cancer. 2007;7:295-308.

6. Bollag G, Hirth P, Tsai J, Zhang J, Ibrahim PN, Cho H, et al. Clinical efficacy of a RAF inhibitor needs broad target blockade in BRAF-mutant melanoma. Nature. 2010;467:596-9.

7. Flaherty KT, Robert C, Hersey P, Nathan P, Garbe C, Milhem M, et al. Improved survival with MEK inhibition in BRAF-mutated melanoma. N Engl J Med. 2012;367:107-14.

8. Hauschild A, Grob JJ, Demidov LV, Jouary T, Gutzmer R, Millward M, et al. Dabrafenib in BRAF-mutated metastatic melanoma: a multicentre, open-label, phase 3 randomised controlled trial. Lancet. 2012;380:358-65.

9. Farrar MA, Alberol-Ila J, Perlmutter RM. Activation of the Raf-1 kinase cascade by coumermycin-induced dimerization. Nature. 1996;383:178-81.

10. Garnett MJ, Rana S, Paterson H, Barford D, Marais R. Wild-type and mutant B-RAF activate C-RAF through distinct mechanisms involving heterodimerization. Mol Cell. 2005;20:963-9.

11. Hatzivassiliou G, Song K, Yen I, Brandhuber BJ, Anderson DJ, Alvarado R, et al. RAF inhibitors prime wild-type RAF to activate the MAPK pathway and enhance growth. Nature. 2010;464:431-5.

12. Heidorn SJ, Milagre C, Whittaker S, Nourry A, Niculescu-Duvas I, Dhomen N, et al. Kinase-dead BRAF and oncogenic RAS cooperate to drive tumor progression through CRAF. Cell. 2010;140:209-21.

13. Luo Z, Tzivion G, Belshaw PJ, Vavvas D, Marshall M, Avruch J. Oligomerization activates c-Raf-1 through a Ras-dependent mechanism. Nature. 1996;383:181-5.

14. Poulikakos PI, Zhang C, Bollag G, Shokat KM, Rosen N. RAF inhibitors transactivate RAF dimers and ERK signalling in cells with wild-type BRAF. Nature. 2010;464:427-30.

15. Rajakulendran T, Sahmi M, Lefrancois M, Sicheri F, Therrien M. A dimerization-dependent mechanism drives RAF catalytic activation. Nature. 2009;461:542-5.
16. Wan PT, Garnett MJ, Roe SM, Lee S, Niculescu-Duvaz D, Good $\mathrm{VM}$, et al. Mechanism of activation of the RAF-ERK signaling pathway by oncogenic mutations of B-RAF. Cell. 2004;116:855-67.

17. Weber CK, Slupsky JR, Kalmes HA, Rapp UR. Active Ras induces heterodimerization of cRaf and BRaf. Cancer Res. 2001;61:3595-8.

18. Wan L, Chen M, Cao J, Dai X, Yin Q, Zhang J, et al. The APC/C E3 ligase complex activator FZR1 restricts BRAF oncogenic function. Cancer Discov. 2017;7:424-41.

19. Haling JR, Sudhamsu J, Yen I, Sideris S, Sandoval W, Phung W, et al. Structure of the BRAF-MEK complex reveals a kinase activity independent role for BRAF in MAPK signaling. Cancer Cell. 2014;26:402-13.

20. Hu J, Stites EC, Yu H, Germino EA, Meharena HS, Stork PJ, et al. Allosteric activation of functionally asymmetric RAF kinase dimers. Cell. 2013;154:1036-46.

21. Desideri E, Cavallo AL, Baccarini M. Alike but different: RAF paralogs and their signaling outputs. Cell. 2015;161:967-70.

22. Jain P, Fierst TM, Han HJ, Smith TE, Vakil A, Storm PB, et al. CRAF gene fusions in pediatric low-grade gliomas define a distinct drug response based on dimerization profiles. Oncogene. 2017;36:6348-58.

23. Palanisamy N, Ateeq B, Kalyana-Sundaram S, Pflueger D, Ramnarayanan K, Shankar S, et al. Rearrangements of the RAF kinase pathway in prostate cancer, gastric cancer and melanoma. Nat Med. 2010;16:793-8.

24. Ross JS, Wang K, Chmielecki J, Gay L, Johnson A, Chudnovsky $\mathrm{J}$, et al. The distribution of BRAF gene fusions in solid tumors and response to targeted therapy. Int J Cancer. 2016;138:881-90.

25. Stransky N, Cerami E, Schalm S, Kim JL, Lengauer C. The landscape of kinase fusions in cancer. Nat Commun. 2014;5:4846.

26. Poulikakos PI, Persaud Y, Janakiraman M, Kong X, Ng C, Moriceau $\mathrm{G}$, et al. RAF inhibitor resistance is mediated by dimerization of aberrantly spliced BRAF(V600E). Nature. 2011;480:387-90.

27. Nan X, Tamguney TM, Collisson EA, Lin LJ, Pitt C, Galeas J, et al. Ras-GTP dimers activate the mitogen-activated protein kinase (MAPK) pathway. Proc Natl Acad Sci USA. 2015;112:7996-8001.

28. Hibino K, Shibata T, Yanagida T, Sako Y. A RasGTP-induced conformational change in C-RAF is essential for accurate molecular recognition. Biophys J. 2009;97:1277-87.

29. Jin T, Lavoie H, Sahmi M, David M, Hilt C, Hammell A, et al. RAF inhibitors promote RAS-RAF interaction by allosterically disrupting RAF autoinhibition. Nat Commun. 2017;8:1211.

30. Nelson DS, Quispel W, Badalian-Very G, van Halteren AG, van den Bos C, Bovee JV, et al. Somatic activating ARAF mutations in Langerhans cell histiocytosis. Blood. 2014;123:3152-5.

31. Chakraborty R, Burke TM, Hampton OA, Zinn DJ, Lim KP, Abhyankar $\mathrm{H}$, et al. Alternative genetic mechanisms of BRAF activation in Langerhans cell histiocytosis. Blood. 2016;128:2533-7.

32. Chen SH, Zhang Y, Van Horn RD, Yin T, Buchanan S, Yadav V, et al. Oncogenic BRAF deletions that function as homodimers and are sensitive to inhibition by RAF dimer inhibitor LY3009120. Cancer Discov. 2016;6:300-15.

33. Estep AL, Palmer C, McCormick F, Rauen KA. Mutation analysis of BRAF, MEK1 and MEK2 in 15 ovarian cancer cell lines: implications for therapy. PLOS ONE. 2007;2:e1279.

34. Foster SA, Whalen DM, Ozen A, Wongchenko MJ, Yin J, Yen I, et al. Activation mechanism of oncogenic deletion mutations in BRAF, EGFR, and HER2. Cancer Cell. 2016;29:477-93.

35. Luker KE, Smith MC, Luker GD, Gammon ST, Piwnica-Worms H, Piwnica-Worms D. Kinetics of regulated protein-protein interactions revealed with firefly luciferase complementation 
imaging in cells and living animals. Proc Natl Acad Sci USA. 2004;101:12288-93.

36. Hu J, Ahuja LG, Meharena HS, Kannan N, Kornev AP, Taylor SS, et al. Kinase regulation by hydrophobic spine assembly in cancer. Mol Cell Biol. 2015;35:264-76.

37. Hu J, Yu H, Kornev AP, Zhao J, Filbert EL, Taylor SS, et al. Mutation that blocks ATP binding creates a pseudokinase stabilizing the scaffolding function of kinase suppressor of Ras, CRAF and BRAF. Proc Natl Acad Sci USA. 2011;108:6067-72.

38. Kornev AP, Haste NM, Taylor SS, Eyck LF. Surface comparison of active and inactive protein kinases identifies a conserved activation mechanism. Proc Natl Acad Sci USA. 2006;103:17783-8.

39. Taylor SS, Kornev AP. Protein kinases: evolution of dynamic regulatory proteins. Trends Biochem Sci. 2011;36:65-77.

40. Shaw AS, Kornev AP, Hu J, Ahuja LG, Taylor SS. Kinases and pseudokinases: lessons from RAF. Mol Cell Biol. 2014;34:1538-46.

41. Taylor SS, Shaw A, Hu J, Meharena HS, Kornev A. Pseudokinases from a structural perspective. Biochem Soc Trans. 2013;41:981-6.

42. Yang J, Wu J, Steichen JM, Kornev AP, Deal MS, Li S, et al. A conserved Glu-Arg salt bridge connects coevolved motifs that define the eukaryotic protein kinase fold. J Mol Biol. 2012;415:666-79.

43. Beenstock J, Mooshayef N, Engelberg D. How do protein kinases take a selfie (autophosphorylate)? Trends Biochem Sci. 2016;41:938-53.

44. Lavoie H, Li JJ, Thevakumaran N, Therrien M, Sicheri F. Dimerization-induced allostery in protein kinase regulation. Trends Biochem Sci. 2014;39:475-86.

45. Valley CC, Arndt-Jovin DJ, Karedla N, Steinkamp MP, Chizhik AI, Hlavacek WS, et al. Enhanced dimerization drives ligandindependent activity of mutant epidermal growth factor receptor in lung cancer. Mol Biol Cell. 2015;26:4087-99.

46. Ritt DA, Monson DM, Specht SI, Morrison DK. Impact of feedback phosphorylation and Raf heterodimerization on normal and mutant B-Raf signaling. Mol Cell Biol. 2010;30:806-19.

47. Cseh B, Doma E, Baccarini M. "RAF" neighborhood: protein-protein interaction in the Raf/Mek/Erk pathway. FEBS Lett. 2014;588:2398-406.

48. Chong H, Vikis HG, Guan KL. Mechanisms of regulating the Raf kinase family. Cell Signal. 2003;15:463-9.

49. Lavoie $H$, Therrien M. Regulation of RAF protein kinases in ERK signalling. Nat Rev Mol Cell Biol. 2015;16:281-98.
50. Fabrini R, De Luca A, Stella L, Mei G, Orioni B, Ciccone S, et al. Monomer-dimer equilibrium in glutathione transferases: a critical re-examination. Biochemistry. 2009;48:10473-82.

51. Roring M, Herr R, Fiala GJ, Heilmann K, Braun S, Eisenhardt $\mathrm{AE}$, et al. Distinct requirement for an intact dimer interface in wild-type, V600E and kinase-dead B-Raf signalling. EMBO J. 2012;31:2629-47.

52. Diedrich B, Rigbolt KT, Roring M, Herr R, Kaeser-Pebernard S, Gretzmeier C, et al. Discrete cytosolic macromolecular BRAF complexes exhibit distinct activities and composition. EMBO J. 2017;36:646-63.

53. Thevakumaran N, Lavoie H, Critton DA, Tebben A, Marinier A, Sicheri F, et al. Crystal structure of a BRAF kinase domain monomer explains basis for allosteric regulation. Nat Struct Mol Biol. 2015;22:37-43.

54. Kumar-Sinha C, Kalyana-Sundaram S, Chinnaiyan AM. Landscape of gene fusions in epithelial cancers: seq and ye shall find. Genome Med. 2015;7:129.

55. Mooz J, Oberoi-Khanuja TK, Harms GS, Wang W, Jaiswal BS, Seshagiri S, et al. Dimerization of the kinase ARAF promotes MAPK pathway activation and cell migration. Sci Signal. 2014;7: ra73.

56. Rebocho AP, Marais R. ARAF acts as a scaffold to stabilize BRAF:CRAF heterodimers. Oncogene. 2013;32:3207-12.

57. Rizos H, Menzies AM, Pupo GM, Carlino MS, Fung C, Hyman J, et al. BRAF inhibitor resistance mechanisms in metastatic melanoma: spectrum and clinical impact. Clin Cancer Res. 2014;20:1965-77.

58. Galabova-Kovacs G, Matzen D, Piazzolla D, Meissl K, Plyushch $\mathrm{T}$, Chen AP, et al. Essential role of B-Raf in ERK activation during extraembryonic development. Proc Natl Acad Sci USA. 2006;103:1325-30.

59. Jesenberger V, Procyk KJ, Ruth J, Schreiber M, Theussl HC, Wagner EF, et al. Protective role of Raf-1 in Salmonella-induced macrophage apoptosis. J Exp Med. 2001;193:353-64.

60. Hu J, Strauch P, Rubtsov A, Donovan EE, Pelanda R, Torres RM. Lsc activity is controlled by oligomerization and regulates integrin adhesion. Mol Immunol. 2008;45:1825-36.

61. Wang X, Boyken SE, Hu J, Xu X, Rimer RP, Shea MA, et al. Calmodulin and $\operatorname{PI}(3,4,5) \mathrm{P}(3)$ cooperatively bind to the Itk pleckstrin homology domain to promote efficient calcium signaling and IL-17A production. Sci Signal. 2014;7:ra74. 